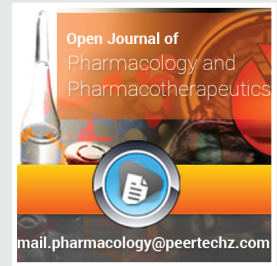

\title{
Open Journal of Pharmacology and Pharmacotherapeutics
}

\section{Ateeq Rahman*, Veikko Uahengo and Daniel Likius}

Faculty of Science, Department of Chemistry and Biochemistry, University of Namibia, Pioneerspark, Private Bag-13301. Windhoek, Namibia

Received: 28 February 2018

Accepted: 25 October 2018

Published: 26 October 2018

*Corresponding author: Ateeq Rahman, Faculty of Science, Department of Chemistry and Biochemistry, University of Namibia, Pioneerspark, Private Bag-13301. Windhoek, Namibia, Email: ateeqr786@yahoo.com

Keywords: Liposome; Drug delivery; Methods; Cell; Carriers

https://www.peertechz.com

Check for updates
Review Article

Mini review on emerging methods of preparation of liposome and its application as Liposome drug delivery

\section{systems}

\section{Introduction}

Drug delivery systems have been envisaged and developed to deliver the drug to the site of action (targeted delivery) to avoid fluctuations in plasma drug levels (controlled release), slow release in recent years to overcome cellular barriers and enzymatic degradation, which impede absorption [1]. Development of new drug molecules is expensive and timeconsuming. Improvement of the safety efficacy ratio of "old" drugs is achieved by individualizing drug therapy, dose titration and therapeutic drug monitoring. Developing drug delivery systems is another attractive option and is being pursued very briskly to improve the safety, reliability [2]. Liposome, microscopic, fluid-filled pouch whose walls are made of layers of phospholipids identical to the phospholipids that make up cell membranes. Liposomes are used to deliver certain vaccines, enzymes, or drugs (e.g., insulin and some cancer drugs) to the body. When used in the delivery of certain cancer drugs, liposomes help to shield healthy cells from the drugs' toxicity and prevent their concentration in vulnerable tissues (e.g., the kidneys, and liver), lessening or eliminating the common side effects of nausea, fatigue, and hair loss [3]. Liposomes are especially effective in treating diseases that affect the phagocytes of the immune system because they tend to accumulate in the phagocytes, which recognize them as foreign invaders. They have also been used experimentally to carry normal genes into a cell in order to replace defective, disease-causing genes [3-5]. Liposomes are occasionally used in cosmetics because of their moisturizing qualities. In some cases liposomes attach to cellular membranes and appear to fuse with them, releasing their contents into the cell

The artificial lipid vesicles (also called liposomes) have been acknowledged andcomprehensively used as delivery vehicles for pharmaceuticals [6], as chemical microreactors [7-10], and as model biomembrane systems [10]. The phospholipid bilayer envelope is a cell-likeboundary applicable for cellular investigations and affordsliposomes a functional scaffold suitable for fundamental cellular functions such as motility and shape change [11], not to mention the aptitude to mimic the biophysical properties of living cells [12]. These "dynamic" behaviors refer to functions such as membrane deformation and actin polymerization whichimpart cell-like kinetic behavior to liposomes [8]. Innovative methodologies to construct improved liposomes for therapeutic delivery have addressed, on one end, biophysical parameters (one common 
example is charge [9]. Which can be manipulated by altering the constituent bilayer phospholipids to better tailor the liposome to the required application. Additional parameters that can and have been manipulated include lamellarity [7], bilayer curvature bilayer fluidity [10-11], as well as surface modification for active or passive targeting approaches [12]. Assembly methods play a key role in defining final liposome characteristics, including encapsulation efficiency and drug release profiles. Hence, recently, the use of synthetic inorganic or polymer nanoparticles to stabilize or support liposomes has emerged as a promising strategy to create hybrid liposomal carriers [13-16]. To date, hundreds of imaging agents and drugs, including fluorophores, nanoparticles, chelating agents, peptides, and proteins, as well as anticancer and antimicrobial agents, vaccines and oligonucleotides, have been integrated into liposomes for a broad range of theranostic applications [17].

From the first liposomal pharmaceutical product-Doxil permitted in 1995 to the latest Marqibo in 2012, there are afewsuccessful liposomal formulations (Table 1). Most of them have to be administrated intravenously due to the degradation of lipids in the gastrointestinal tract. However, some recent formulations such as Arikace (Table 2), can be intravenously injected or inhaled as aerosols. Apart from a broadened range of drugs being investigated for liposomal formulations, new strategies such as environmental sensitivity and combination therapy have been applied to the development process to achieve better efficacy. Furthermore, liposomes could be fruitfully applied to areas other than cancer therapy, such as vaccines [18-20]. Development of liposomal drugs: a typical example of doxorubicin. Doxorubicin, a kind of anthracyclines, is a potent and comprehensive spectrum anti-cancer drug and has been applied as a "first line" medicine in cancer therapy [21]. Two main mechanisms of action are involved for the drug: (1) it inhibits DNA and RNA synthesis by implanting in base pairs of DNA strands, thus preventing the replication and transcription in rapidly growing cancer cells; (2) it inhibits the enzyme topoisomerase II, which is ansupplementary way for blocking DNA transcription and replication. Nevertheless, the positively charged doxorubicin is also of high affinity to negatively charged cardiolipin, which is abundant in heart tissue [22]. This damage results in the dangerous cumulative dose-dependent cardiotoxicity (i.e. irreversible congestive

Table1: Approved Liposomal Formulations.

\begin{tabular}{|c|c|c|c|c|c|}
\hline Drug & Product name & Type & Lipid Composition & $\begin{array}{c}\text { Route of } \\
\text { Administration }\end{array}$ & Approved treatment \\
\hline Amphotericin B & Ambisome & Liposome & HSPC,DSPG and choleserol & Intravenous & Sever fungal infections \\
\hline \multirow[t]{3}{*}{ Doxorubicin } & Myocet & liposome & EPC and cholesterol & Intravenous & metastatic breast cancer \\
\hline & Doxil & pegylated limposome & HSPC,cholesterol and DSPE-PEG2000 & Intravenous & kaposi's sarcoma,ovarian and breast cancer \\
\hline & Lipo-dox & pegylated limposome & DSPC,cholesterol and DSPE-PEG2000 & Intravenous & kaposi's sarcoma,ovarian and breast cancer \\
\hline Daunorubicin & Daunoxome & Liposome & DSPC and cholesterol & Intravenous & Blood cancer \\
\hline Verteporfin & Visudyne & Liposome & EPG and DMPC & Intravenous & Age-related molecular degeneration \\
\hline Cytarabine & Depocyt & liposome & DPOC, DPPG, cholesterol and triolein & Spinal & Neoplastic meningitis and lymphomatous meningitis \\
\hline Morphine sulfate & Depodur & liposome & DPOC, DPPG, cholesterol and triolein & Epidural & Pain \\
\hline Vincristine sulfate & Marqibo & Liposome & Egg sphingomyelin and cholesterol & Intravenous & Acute lymphoblastic leukemia \\
\hline
\end{tabular}

Table 2: Liposomal formulations in clinical trials.

\begin{tabular}{|c|c|c|c|c|c|}
\hline Drug & $\begin{array}{l}\text { Product } \\
\text { name }\end{array}$ & Lipid Composition & $\begin{array}{c}\text { Route of } \\
\text { administration }\end{array}$ & Treatment under investigation & $\begin{array}{c}\text { Trial } \\
\text { phase }\end{array}$ \\
\hline \multirow[t]{2}{*}{ Paclitaxel } & LEP-ETU & DOPC, cholesterol and cardiolipin & Intravenous & Ovarian, breast and lung cancers & 1 \\
\hline & Endo TAG-1 & DOTAP and DOPC & Intravenous & Anti-angiogenesis, breast and pancreatic cancers & II \\
\hline Doxorubicin & ThermoDox & DPPC,MSPC and DSPE-PEG2000 & Intravenous & Non-resectable hepatocellular carcinoma & III \\
\hline \multirow[t]{3}{*}{$\begin{array}{l}\text { Cisplatin and its } \\
\text { analog }\end{array}$} & SPI-077 & HSPC, cholesterol and DSPE-mPEG & Intravenous & Lung, head and neck cancers & $\mathrm{I} / \mathrm{II}$ \\
\hline & Lipoplatin & $\begin{array}{l}\text { SPC, DPPG, cholesterol and DSPE- } \\
\text { mPEG }\end{array}$ & Intravenous & $\begin{array}{l}\text { Pancreatic cancer, head and neck cancer, mesothelioma, breast cancer, } \\
\text { gastric cancer and non-small-cell lung cancer. }\end{array}$ & III \\
\hline & Aroplatin & DMPC and DMPG & $\begin{array}{l}\text { Intrapleural/ } \\
\text { intravenous }\end{array}$ & Malignant pleural mesothelioma and advanced colorectal carcinoma & II \\
\hline Mitoxantrone & LEM-ETU & DPOC, cholesterol and cardiolipin & Intravenous & Leukemia, breast, stomach, liver and ovarian cancers & I \\
\hline Topotecan & INX-0076 & Egg sphingomyelin and cholesterol & Intravenous & Advanced solid tumors & I \\
\hline Vinorelbine & INX-0125 & Egg sphingomyelin and cholesterol & Intravenous & Brest, colon and lung cancers & 1 \\
\hline Lurtotecan & OSI-211 & HSPC and cholesterol & Intravenous & Ovarian, head and neck cancers & II \\
\hline Amikacin & Arikace & DPPC and cholesterol & $\begin{array}{l}\text { Inhaled as } \\
\text { aerosol }\end{array}$ & Lung infection & III \\
\hline $\begin{array}{l}\text { BLP25 } \\
\text { lipopeptide }\end{array}$ & Stimuvax & $\begin{array}{l}\text { Monophosphoryl lipid A, cholesterol. } \\
\text { DMPG and DPPC }\end{array}$ & Subcutaneous & Non-small-cell lung carcinoma & III \\
\hline
\end{tabular}


failure), which significantly limits the tolerable dose range of doxorubicin. Further side effects of doxorubicin include severe myelosuppression, nausea and vomiting and mucocutaneous toxicities [23]. Consequently, liposomal formulation is proposed to overcome these toxicities. Initially, liposomal doxorubicin was prepared to be negatively charged, mediumsize oligolamellar liposomes, in which the drug was passively entrapped by the lipid hydration method [24]. However, this formulation failed in following clinical trials mainly due to the rapid drug release and clearance by reticuloendothelial system in vivo. "Remote loading" was then used to improve the drug loading proficiency and formulation stability, bringing about Myocet and Doxil in which doxorubicin was loaded by a $\mathrm{pH}$ orammonium gradient, respectively. The morphology and structure of Doxil is shown in fiugre 1. A major advancement of Doxil over Myocet is the coating with PEG, which significantly improves its pharmacokinetic profile. So in a pharmacokinetic study of doxorubicin loaded liposomes, free doxorubicin had an elimination half-life of $0.2 \mathrm{~h}$ and an area under the plasma concentration time curve (AUC) of $3.81 \mathrm{mg} \mathrm{h} / \mathrm{ml}$, compared with $2 \mathrm{e} 3 \mathrm{hand} 46 \mathrm{mg} \mathrm{h} / \mathrm{ml}$ for Myocet and with a further increase to $41 \mathrm{e} 70 \mathrm{~h}$ and $902 \mathrm{mg} \mathrm{h} / \mathrm{ml}$ for Doxil [25]. Both Myocet and Doxil considerably reduce the toxic effects of doxorubicin. In a Phase III comparison of free doxorubicin with Myocet, patients. Table 1 represents approved liposomal formulations [26] (Table 2). Liposomal formulations in clinical trials [27].

Advance of liposomal drugs: A characteristic example of doxorubicin Doxorubicin, a kind of anthracyclines, is a potent and widespectrum anti-cancer drug and has been used as a "firstline" medicine in cancer therapy [21]. Two main mechanisms of action are involved for the drug: (1) it inhibits DNA and RNA synthesis by inserting in base pairs of DNA strands, thus inhibiting the replication and transcription in swiftly growing cancer cells; (2) it inhibits the enzyme topoisomerase II, which is a surplus way for blocking DNA transcription and replication. Conversely, the positively charged doxorubicin is also of high affinity to negatively charged cardiolipin, which is plentiful in heart tissue [22]. This damage results in the dangerous cumulative dose-dependent cardiotoxicity (i.e. irreversible congestive failure), which substantially limits the tolerable dose range of doxorubicin. Other side effects of doxorubicin include severe myelosuppression, nausea and vomiting and mucocutaneous toxicities [23]. Therefore, liposomal formulation is proposed to overcome these toxicities. Originally, liposomal doxorubicin was prepared to be

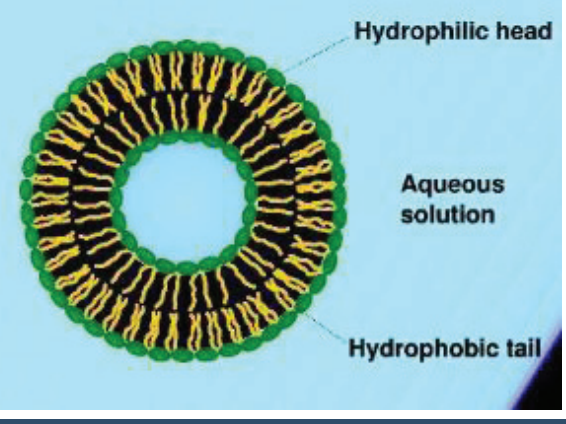

Figure 1: Liposome image [1]. negatively charged, medium-size oligolamellar liposomes, in which the drug was passively entrapped by the lipid hydration method [24]. Hence,this formulation failed in following clinical trials mainly due to the rapid drug release and clearance by reticuloendothelial system in vivo. "Remote loading" was then used to improve the drug loading efficiency and formulation stability, bringing about Myocet and Doxil in which doxorubicin was loaded by a $\mathrm{pH}$ orammonium gradient, respectively. The morphology and structure of Doxil is shown in figure 1. A major advancement of Doxil over Myocet is the coating with PEG, which considerably improves its pharmacokinetic profile. So in a pharmacokinetic study of doxorubicin loaded liposomes, free doxorubicin had an elimination half-life of $0.2 \mathrm{~h}$ and an area under the plasma concentratione time curve (AUC) of $3.81 \mathrm{mg}$ $\mathrm{h} / \mathrm{ml}$, compared with $2 \mathrm{e} 3 \mathrm{hand} 46 \mathrm{mg} \mathrm{h} / \mathrm{ml}$ for Myocet and with a further increase to $41 \mathrm{e} 70 \mathrm{~h}$ and $902 \mathrm{mg} \mathrm{h} / \mathrm{ml}$ for Doxil [25]. Both Myocet and Doxil considerably reduces the toxic effects of doxorubicin. In a Phase III comparison of free doxorubicin with Myocet, patients treated with Myocet had low incidence of cardiac events (13\%vs. $29 \%$ ), mucositis/stomatitis ( $8.6 \%$ vs. $11.9 \%$ ), and nausea/vomiting (12.3\% vs. $20.3 \%$ ) [26]. Analogous results were found inanother Phase III trial of Doxil, in which the reduction of cardiotoxicity $(3.9 \%$ vs. $18.8 \%)$, neutropenia ( $4 \%$ vs. $10 \%$ ), vomiting( $19 \%$ vs. $31 \%$ ), and alopecia ( $20 \%$ vs. $66 \%$ ) were found [27]. Nevertheless, equivalent survival rates between liposomes andfree drugs were found in these studies, signifying the advantage of Myocet and Doxil lay only in the reduction of toxicities.

Vincristine, like the other vinca alkaloids, is very active against many of the lymphoid malignancies, including forceful non-Hodgkin's lymphoma and all [28]. In adults with ALL, vincristine remains an integral constituent of induction chemotherapy regimens [29-32]. Vincristine acts by binding to tubulin during active mitosis, resultant in microtubule depolymerization and metaphase arrest, important to apoptosis [33-34]. Nevertheless, vincristine also binds to neuronal tubulin, disrupts axonal microtubules, and thus causes severe neurotoxicity, which has led to the clinical practice of capping the total dose of vincristine to $2.0 \mathrm{mg}$ regardless of body surface area, potentially reducing clinical effectiveness. The high affinity of vincristine to both mitotic and neuronal microtubules suggests that it may be difficult to prevent neurotoxicity without reducing clinical efficacy [35].

Vincristine is an imperative and active drug in first-line therapy of all as well as in the treatment of relapsed disease [36]. Its administration is generally limited to weekly injections, usually for 2 months or less in most regimens because of severe peripheral neuropathy, including autonomic neuropathy that often leads to intestinal complications. Since of its narrow therapeutic index, the maximal antileukemia activity of free vincristine is not realized [33]. Thus, an approach to growing the dose intensity of vincristine while decreasing its toxicity through the use of liposome encapsulation technology has been considered.

Liposome-encapsulated vincristine: Vincristine is a vinca alkaloid with activity against a broad range of cancers, including Hodgkin's and non-Hodgkin's lymphoma, chronic 
lymphocytic leukemia, all, and a variety of solid tumors. Vincristine exerts its cytotoxic activity by attributing to the increasing end of microtubules and stopping their assembly, arresting cell growth in metaphase. Therefore, as a cell-cycle specific cytotoxic drug, prolonging exposure time to the drug is anticipated to be therapeutically beneficial; perhaps even more so than prolonged exposure to anthracyclines which do not act in a cell cycle-specific manner. In leukemia cell lines, exposure to vincristine from one to 72 hours resulted in a 105-fold reduction in the drug concentration wanted to cause $50 \%$ cytotoxicity (IC50), associated with only a 40-fold reduction in the $\mathrm{IC} 50$ for doxorubicin following elongated exposure to the same extent [37-38]. Pharmacokinetic data with free vincristine comprehensive rapid tissue binding, low serum concentrations after intravenous administration, and extensive tissue spreading in vivo [39-40]. Limiting its potential therapeutic efficacy. Although, continuous intravenous infusion of vincristine has been inspected as a way to increase drug exposure, noteworthy neurotoxicity still occurred [41] Furthermore, conventional vincristine is limited by significant peripheral and central nervous system neurotoxicity, which occurs at doses higher than $1.4 \mathrm{mg} / \mathrm{m}^{2}$. Therefore, it was hypothesized that if liposomal encapsulation and delivery results in higher levels of drug at tumor sites for longer periods of time, greatly developed efficacy may be expected with a cell cycle-specific drug like vincristine [31-38], and retention of drug in the liposome would result in lower drug concentrations in tissues where toxicity occurs, containing the peripheral and central nervous systems [33]. Preclinical development of liposome-encapsulated formulations of vincristine: The initial investigations of liposomal encapsulation of vincristine failed to demonstrate a therapeutic improvement over free vincristine sulfate in murine leukemia models [42]. Indeed, the low solubility of vincristine in aqueous solution at physiologic $\mathrm{pH}$ and its relatively high permeability to membranes, resulting in poor retention of drug in the liposome, initially presented significant limitations to improvement of a stable liposomal vincristine formulation. Subsequent work identified that transmembrane $\mathrm{pH}$ gradients, with the inside of the vesicle being acidic, can result in considerably improved trapping of drug in the liposome [43-45]. Additionally, maintenance of the $\mathrm{pH}$ gradient is affected, at least in part, by the lipid composition of the liposome [46]. Developing such transmembrane $\mathrm{pH}$ gradient drug uptake processes, almost $100 \%$ trapping of vincristine was achieved inside egg phosphatidylcholine/ cholesterol and distearoylphosphatidylcholine (DSPC)/ cholesterol liposomes, when the internal $\mathrm{pH}$ of the liposome was lowered to 4.0 [47]. However, while both DSPC/cholesterol and egg phosphatidylcholine/cholesterol preparations had similar vincristine tricking efficiency, the $\mathrm{pH}$ gradient across the membrane was considerably more sustained in DSPC/ cholesterol vesicles with better maintenance of vincristine in the liposome [47], resulting in a longer drug circulation time and enhanced tumor delivery and antitumor activity compared with free vincristine. [47-48]. In murine models of lymphocytic leukemia, the DSPC/cholesterol liposome formulation showed greater antitumor efficacy compared with either free vincristine or the egg phosphatidylcholine/cholesterol formulation [4849] and the LD50 (median lethal dose) was significantly higher with DSPC/cholesterol liposome-encapsulated vincristine formulation (LD50 $4.8 \mathrm{mg} / \mathrm{kg}$ ) compared with free vincristine (LD50 $1.9 \mathrm{mg} / \mathrm{kg}$ ), indicating that liposomal encapsulation increased drug delivery with less toxicity $[46,47]$.

Preclinical models also established that vincristine encapsulated in sphingomyelin/cholesterol liposomes accumulated preferentially at tumor sites, which associated with antitumor activity [50-52]. Moreover, this was related to liposomal extravasation into the tumor rather than uptake by tumor cells of free vincristine that had been free from liposomes in the circulation [51]. In a murine model with human breast cancer xenografts, sphingomyelin/cholesterol liposome-encapsulated vincristine resulted in targeted delivery of the drug, with a fourfold intensification in concentration of drug in tumor tissue and a three-fold increase in bone marrow, with maintenance of substantial tissue drug concentrations for several days compared with free vincristine, and without increased toxicity [52]. The antitumor efficacy of sphingomyelin/cholesterol liposome-encapsulated vincristine has also been confirmed in several preclinical murine and human tumor xenograft models, signifying several cancer types [50-52,54], including human [33]. The aggregate of the above studies supports the utility of encapsulating vincristine in sphingomyelin/cholesterol liposomes to increase drug delivery while limiting release in the central blood compartment to decrease drug toxicity. In 2012, Owellan et al [34], reported an improved method for active loading of doxorubicin into the HaT formulation based on a copper (II) gradient (HaT-II) [53]. HaT-II showed improved in vitro stability at $37^{\circ} \mathrm{C}$, together with a faster drug release rate at $41^{\circ} \mathrm{C}$ in the presence of serum when associated with LTSL [53]. In comparison with LTSL, HaT-II showed a 2.5-fold longer blood circulation time in mice and a 2.0-fold increase in drug delivery to the heated tumor [76]. This resulted in improved antitumor efficacy [54-55]. In 2013, Park et al reported another alleviated formulation composed of DPPC, DSPEPEG2000, cholesterol, and fatty acid-conjugated elastin-like polypeptide 55:2:15:0.4125 (mol/mol) (STL) with encapsulated doxorubicin (Table 1) [55]. Pharmacokinetic studies in mice showed plasma half-lives of 2.03 hours and 0.92 hours for doxorubicin encapsulated in STL and LTSL [55], respectively. In combination with high intensity focused ultrasound, STL achieved significantlybetter tumor growth delay 7 days after injection when compared with LTSL [55]. Doxorubicin and manganese (II) form a stable complex [56], with the paramagnetic manganese (II) serving as an MRI contrast agent. Thus the release kinetics are the same for the contrast agent and the drug, agreeing for correlation between change in $\mathrm{T} 1$ relaxation time (determined by MRI) and amount of doxorubicin (determined by high-performance liquid chromatography) [57]. Using this strategy, it was possible to show that release of doxorubicin was heterogeneously scattered in the tumor model, and that LTSL administered during hyperthermia had the greatest antitumor effect when compared with other administration strategies. Docetaxel is a semi-synthetic microtubule disrupting anticancer drug indicated for the treatment of breast, prostate, non-small-cell lung, head and neck, and gastric cancers [5864]. Standard every 3-week dosages range from 60 to 100 $\mathrm{mg} / \mathrm{m} 2$, with $75 \mathrm{mg} / \mathrm{m} 2$ being a frequently used dose in these 
diseases. Dose-limiting toxicities (DLTs) are myelosuppression and neuropathy, among others. For example, at a dose of 100 $\mathrm{mg} / \mathrm{m} 2$, docetaxel causes grade 4 neutropenia in $75-86 \%$ of patients [65]. Liposomal doxorubicin has been explored, both as monotherapy and in combination with other agents, for the treatment of several malignancies [66], including breast cancer [67-68], and platinum-refractory ovarian cancer [69]. Liposomal doxorubicin (Doxil R) is currently used in the treatment for refractory ovarian cancer and AIDS-related Kaposi's sarcoma [70-71]. Decreased incidence or severity of toxicities compared with free doxorubicin, including relative cardiotoxicity, has been reported in some studies [67-68], supporting the hypothesis of improved safety provided by liposome encapsulation of the active agent. Another approved liposomal anticancer agent, DaunoXomeR (liposomal daunorubicin), is used in the treatment for AIDS-related Kaposi's sarcoma [69].

\section{Methods for preparation of liposomes}

An important parameter to consider when addressing the formation process of liposomes is the rigidity of the bilayer. Hydrated-single component phospholipid bilayers can be in a liquid-crystalline ('fluid') state or in a gel state. By increasing the temperature, the gel state bilayer melts and is converted into the liquid state [70]. This occurs at a temperature known as the transition temperature (Tc). The Tc of a bilayer depends on:

\section{Acyl chain length.}

\section{Degree of saturation.}

3. Polar head group.

The Tc can vary between $150 \mathrm{C}$ for egg yolk phosphatidylcholine (high degree of unsaturation) to over $500 \mathrm{C}$ for fully saturated distearolyl phosphatidylcholine (DSPC) [71-72].The raw material for liposome formation depends on the intended use of the liposome. Numerous companies supply reasonable grade and priced lipids which usually contain at least 98\%phospholipid and less than $1 \%$ lysophospholipid, low endotoxin and microbial load and trace metals. It is up to the individual investigator to purify the lipid to acceptable standards [73].

There are five main groups of phospholipids that are available which is used for liposome preparation [73].

\section{Phospholipid from natural sources.}

2. Phospholipid modified from natural sources.

3. Semi-synthetic phospholipid.

4. Fully-synthetic phospholipid,

5. Phospholipid with non-natural head groups.

Phosphatidylcholine (PC), phosphatidylenolamine (PE) and phosphatidyserine (PS) are commonly used phospholipids for liposome preparation. Cholesterol can be added to the bilayer mixture to reduce the permeability of fluid crystalline state bilayers.

There are many different strategies for the preparation of liposomes, which can be classified into 3 main groups.

\section{Mechanical methods}

A. Film method: The original method of Bangham et al [74], is still the simplest procedure for the liposome formation but is limited because of its low encapsulation efficiency. This technique produces liposomes by hydrating thin lipid films deposited from an organic solution on a glass wall by shaking at temperatures above the Tc. The solvent is removed at reduced pressure in a rotary evaporator. The dry film of lipids which has been deposited onto the wall of a round-bottom flask is hydrated by adding a buffer with a water soluble marker. As the lipid becomes hydrated and starts to form into closed vesicles only a small amount of the solute becomes entrapped. This method yields a heterogeneous sized population of MLVs over $1 \mu \mathrm{m}$ in diameter. Further procedures must be employed to achieve a homogeneous population, which will be discussed later [75].

B. Ultrasonication method: Ultrasonication of an aqueous dispersion of phospholipids with a strong bath sonicator or a probe sonicator will usually yield SUVs with diameters down to $15-25 \mathrm{~nm}$.

\section{Methods based on replacement of organic solvent}

A. Reverse-phase evaporation: In this method, several phospholipids (pure/mixed with cholesterol) can be used. The lipid mixture is added to a round bottom flask and the solvent is removed under reduced pressure by a rotary evaporator [75]. The system is purged with nitrogen and the lipids are re-dissolved in the organic phase. This is the phase that the reverse phase vesicles will form. Diethly ether and isopropyl ether are the usual solvents of choice [71].

After the lipids are re-dissolved in this phase the aqueous phase (contains compound to be encapsulated) is added. The system is kept under continuous nitrogen and the two-phase system is sonicated until the mixture becomes a clear onephase dispersion. The mixture is then placed on the rotary evaporator and the organic solvent removed until a gel is formed. Non-encapsulated material is removed.The resulting liposomes are called Reverse-phase evaporation vesicles (REV). The large unilamellar and oligolamellar vesicles formed have the ability to encapsulate large macromolecular vesicles with high proficiency [71].

B. Ether vaporisation method: In ether vaporisation method a mixture of lipids in an organic solvent (diethyl ether, ethanol, etc). Which is slowly injected into a warm aqueous solution. This results in osmotic ally active, unilamellar vesicles with a well-defined size distribution and high volume trapping efficiency (about ten times that of sonicated and hand shaken preparations [72]. 


\section{Methods based on size transformation or fusion of pre- formed vesicles}

A. Freeze-thaw extrusion method: Liposomes formed by the film method are vortexed with the solute to be entrapped until the entire film is suspended and the resulting MLVs are frozen in a dry ice/acetone bath, thawed in lukewarm water and vortexed again [73]. After two additional cycles of freezethaw and vortexing the sample is extruded three times. This is followed by six freeze-thaw cycles and an additional eight extrusions. The resulting liposomes are called large unilamellar vesicles by extrusion technique (LUVET) and they typically contain internal solute concentrations which are much higher than the external solute concentration's which have entrapment ratios greater than one [6]. Proteins can be effectively encapsulated using this technique [74].

B. The dehydration-rehydration method: This method begins with empty buffer containing SUVs (handshaken MLVs can be also be used but are usually not preferred). These are mixed with the component to be entrapped, after which they are dried. Freeze-drying is often the method of choice but other methods such as by vacuum or under a stream of nitrogen can be used. The vesicles are then rehydrated [7-74]. A mechanism has been proposed whereby as the vesicles become more concentrated during dehydration, they flatten and fuse forming multilamellar planes where the solute is sandwiched. Therefore on hydration, larger vesicles are formed. This technique is mild and simple, the main limitation being the heterogeneity of the size of the size of the liposomes [75].

Sizing of the liposomes: Size characteristics of liposomes have a major effect on their fate. Therefore, liposome production procedures must generate predictable and reproducible particle size distributions within a certain size range.Sizing of liposomes (if the population is heterogeneous) is usually performed by sequential extrusion at relatively low pressures through polycarbonate membranes [75]. It's easily, reproducible, no detectable degradation of the phospholipids takes place and it can double the encapsulation efficiency of the liposome preparation. Membranes of pore size $\sim 0.2 \mu \mathrm{m}$ will yield liposomes of $\sim 0.27 \mu \mathrm{m}[76]$. Gel chromatography can also be used to size liposomes but more typically used to remove un-encapsulated components by separation [2]. Sonication is another process that is widely applied when sizing liposomes. Probe sonication is used rather than bath sonication and it produces small unilamellar vesicles of $20 \mathrm{~nm}$.

There are many disadvantages associated with this technique:

1. Exclusion of oxygen is difficult which results in peroxidation reactions.

2. Titanium probes shed metal particles resulting in contamination.

3. They can generate aerosol's which excludes them with from use with certain agents.

These problems can be avoided with the use of bath sonicators but reproducible results are difficult because of the number of varying parameters associated with such baths (level and temperature of water, position ofliposome in the bath, etc.) [75-76].

Liposomes in immune assays: The liposome immune assay system is usually based on membrane immune chemistry and the release of a detectable marker. In an enzyme linked immune sorbent assay (ELISA) the enzyme label generates a measurable amount of product which is proportional to the unknown concentration of an antigen. Liposomes are artificially prepared vesicles made of lipid bilayer. Liposomes can be filled with drugs, composite materials and used to deliver drugs for cancer and other diseases [77]. Liposomes can be composed of naturally-derived phospholipids with mixed lipid chains (like egg phosphatidylethanolamine) or other surfactants [78].

Subsequently the research conducted by new et. al. [79], much interest have been centered on the use of liposomes as a drug carrier for Amp B in the treatment of several systemic fungal and parasitic infections. It was shown that L-Amp B was as effective as free Amp B in experimental histoplasmosis [80], and cryptococcosis [81], but much less toxic [82]. LopezBeresteinet al. [82], carried out extensive studies on the use of L-Amp B in systemic candidiasis and paved the way for its clinical use. They used multilamellar liposomes prepared form dimyristoyl phosphatidylcholine (DMPC) and dimyristoyl phosphatidylglycerolin a 7:3 molar ratio. Hopferet.al. [83], observed that the lipid composition of the liposomes played a major role in L-Amp B activity. The presence of a sterol component (like ergosterol and cholesterol) in liposomes decreased the antifungal activity by almost 50 -fold. However, Tremblay Gomdal et al and Szokaet. Al. [84-87], have found that the incorporation of cholesterol in liposomes did not result in any loss of activity. It was postulated that in multilamellar vesicles (MLV), only about $10 \%$ of the lipid is on the external monolayer and the transfer of Amp B from the internal lamellae to the fungal cell cannot take place readily. While small unilamellar vesicles (SUV) containing cholesterol have about $50-60 \%$ of the lipids on the monolayer accounting for better transfer of Amp B. With this concept, two SUV formulations were developed. Negatively charged small unilamellar vesicles made from hydrogenated soya phosphatidylcholine (SPC), cholesterol and distearoyl phosphatidyl glycerol (DSPG) in 2:1:0.8 molar ratios were tested in murine candidiasis and cryptococcosis [85-87]. The efficacy was found to be comparable with conventional Amp $\mathrm{B}$ on an equal dose basis. The other formulation is positively charged, prepared from SPC, cholesterol and stearyl amine in 4:3:1 molar ratio [88]. Liposomes can be prepared by disrupting biological membranes, for example by sonication. Liposomes can be composed of naturally-derived phospholipids with mixed lipid chains (like egg phosphatidylethanolamine) or other surfactants. Liposomes should not be confused with micelles and reverse micelles composed of monolayer [89].

\section{Liposomes act as carrier radioactive compounds as ra- dio tracers}

In addition, liposomes can be used to carry radioactive compounds as radiotracers can be linked to multiple locations 
in liposomes [90]. One option is the hydrated compartment inside the liposome, another the lipid core into which especially hydrophobic conjugates can be attached, and the third option is the outer lipid leaflet where molecules can be bound by covalent linkage. Delivery of agents to the reticuloendothelial system (RES) is easily achieved, since most conventional liposomes are trapped by the RES. For the purpose of delivery of agents to target organs other than RES, long-circulating liposomes have been developed by modifying the liposomal surface [91]. Understanding of the in vivo dynamics of liposome-carried agents is required for the evaluation of the bio-availability of drugs encapsulated in liposomes. Amphotericin B (Amp B) remains the drug of choice in most systemic mycoses and also as a second line treatment for Kalaazar [91]. However, its toxic effects often limit its use. Although the liposome delivery system has been tried for several drugs, only a few have been used in patients due to the slow development of necessary large-scale pharmaceutical procedures. As compared to conventional Amp $\mathrm{B}$, Fungisome are infused over a much shorter period requiring a smaller volume and no pre-medication. It was found to be safe in patients who had developed serious unacceptable toxicity with conventional Amp B. In renal transplant patients, fungisome did not produce any nephrotoxicity. Fungi some are effective in fungal infections resistant to fluconazole, conventional Amp B and in virgin and resistant cases of visceral leishmaniasis. Amphotericin B (Amp B) is a polyene macrolide antibiotic that is widely used for the treatment of systemic fungal infections. Disseminated fungal infections are a major cause of morbidity and mortality in patients with leukaemia receiving chemotherapy and in a variety of immuno-deficiency diseases [89]. The majority of these infections are caused by the species of Candida and Aspergillus. Despite the development of new classes of antifungal agents, Amp B remains the drug of choice. Its antimicrobial activity results from its ability to bind to the sterol component of the cell membrane, leading to the formation of transmembrane pores that allow the leakage of vital cellular constituents. Amp B binds preferentially to ergosterol, a major component of the fungal cell wall. Unfortunately, the drug also interacts with cholesterol in mammalian membrane, which probably is the basis for its profound acute and chronic toxicity. Approximately $20-50 \%$ patients treated with Amp B develop acute infusion-related reactions such as fever, chills, nausea and vomiting [90]. This is in spite of the liberal use of premedication'sintended to prevent such side effects. Clements and Peaco [91], observed that nephrotoxicity is one of the most central chronic toxicities associated with Amp $B$ usage because of its potential limiting effect on the total course of therapy. Nephrotoxicity is present in about 60$83 \%$ of patients. Additional significant encountered chronic toxicity is electrolyte disturbance secondary to renal wasting of potassium and magnesium. Ninety percent of patients on Amp B treatment require potassium supplementation [92].

Preparation of Multi-lamellar Liposomes: The methodology for preparation of MLVs is to use well characterized lipids in order to produce well defined liposomes [93-96]. Equally significant is the selection of bilayer components for toxicity and for shelf life optimization. The lipids normally used are the unsaturated egg phosphatidylcholine (PC), phosphatidic acid (PA), phosphatidylglycerol (PG), and the saturated lipids DMPC, dipalmitoylphosphatidylcholine (DPPC), dipalmitoylphosphatidic acid (DPPA), and dipalmitoylphosphatidylglycerol (DMPG). Stearylamine is used when cationic liposomes are favored; and natural acidic lipids, such as phosphatidylserine (PS), PG, phosphatidylinositol (PI), $\mathrm{PA}$, and cardiolipin (CL) are added when anionic liposomes are desired, while cholesterol is often involved to stabilize the bilayer. Small amounts of antioxidants such as $\alpha$-tocopherol or $\beta$-hydroxytoluidine (BHT) are included when polyunsaturated neutral lipids are used [96].

Preparation of Lipid for hydration: Lasic et al [97] while preparing liposomes with mixed lipid composition, the lipids must first get dissolved and mixed in an organic solvent to confirm a homogeneous mixture of lipids. In general this process is carried out using chloroform or chloroform:methanol mixtures. The intent is to obtain a clear lipid solution for complete mixing of lipids. Normally lipid solutions are prepared at $10-20 \mathrm{mg}$ lipid/ml organic solvent, although greater concentrations may be used if the lipid solubility and mixing arestandard. When the lipids are thoroughly mixed in the organic solvent, the solvent is removed to yield a lipid film. For small volumes of organic solvent $(<1 \mathrm{~mL})$, the solvent may be evaporated using a dry nitrogen or argon stream in a fume hood. For larger volumes, the organic solvent should be removed by rotary evaporation yielding a thin lipid film on the sides of a round bottom flask [97]. The lipid film is comprehensively dried to remove residual organic solvent by placing the vial or flask on a vacuum pump overnight. If the use of chloroform is objectionable, an alternative is to dissolve the lipids in t-butanol or cyclohexane. The lipid solution is transferred to containers and frozen by placing the containers on a block of dry ice or swirling the container in a dry iceacetone or alcohol (ethanol or methanol) bath. Care must be taken when using the bath procedure that the container can withstand sudden temperature changes without cracking. After freezing completely, the frozen lipid cake is placed on a vacuum pump and lyophilized until dry (1-3 days depending on volume). The thickness of the lipid cake should be no more than the diameter of the container being used for lyophilization.Dry lipid films or cakes can be removed from the vacuum pump, the container close tightly and taped, and stored frozen until ready to hydrate. Basu.S et.,al., [98] proposed general methods to prepare MLV (Figure 2).

\section{A general protocol to prepare MLV is as follows}

1. Laughrey eta at et.al [99]. Discussed the preparation of lipid component. A suitable solution of the lipid component in a pear-shaped flask (lipid concentrations between 5 and 50 mMin either chloroform or in chloroform-methanol (3_1, v/v), and filter the mixture to remove minor insoluble components or ultra-filter to reduce or eliminate pyrogens.

2. Employing a rotary evaporator, remove the solvent, while maintaining a temperatureof $\sim 40^{\circ} \mathrm{C}$ in a water bath under negative pressure. Other methods of drying include spray drying and lyophilization [99]. Traces of organic solvents are removed employing a vacuum pump, normally overnight at 


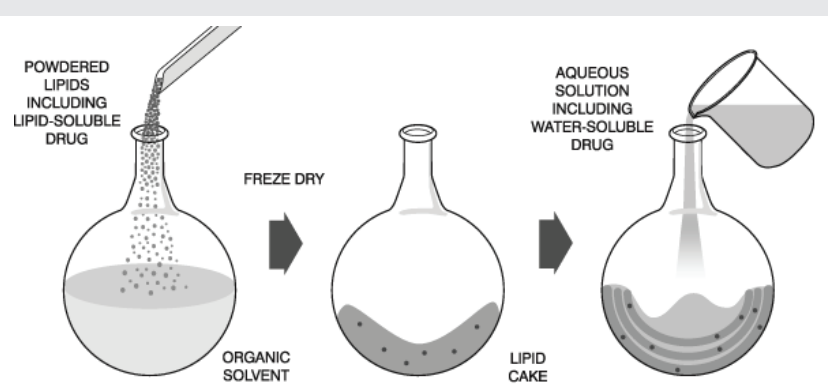

CAKE
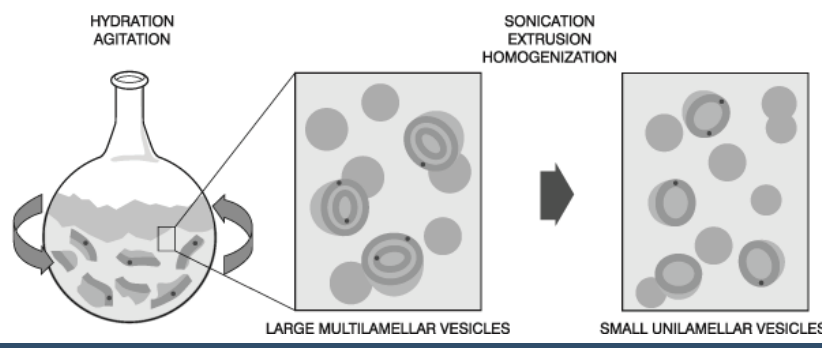

Figure 2: Preparation of lipid [118]

pressures below milliTorr $(\sim 0.1 \mathrm{~Pa})$. Alternatively, the sample may be dried under a very low vacuum $(<50 \mu \mathrm{mol} / \mathrm{mg})$ for $1-2$ $\mathrm{h}$ in a desiccator with drierite ${ }^{\mathrm{TM}}$ (Fisher Scientific, Malvern, PA) [98].

3. Subsequent to drying, $100 \mu \mathrm{L}$ of $0.5 \mathrm{~mm}$ glass beads are added to the $10-\mathrm{mL}$ flask containing the dried lipid mixture, and hydration fluid ( $0.308 \mathrm{M}$ glucose), which is equal to the final volume of the liposome suspension, is added. Typically, the volume of hydration fluid used is determined by the amount of liposomal phospholipid and is usually in millimolars with respect to the hydration fluid [98].

4. Vortex mixing the flask for 1-2 min causes all of the dried lipid from the flask to be dispensed into the hydration fluid. Alternative hydration mediums are distilled water, buffer solution, saline, or non-electrolytes such as a sugar solution. For an in vivo preparation, physiological osmolality $(290 \mathrm{mosmol} / \mathrm{kg})$ is recommended and can be achieved using $0.6 \%$ saline, $5 \%$ dextrose, or $10 \%$ sucrose solution. MLVs of tens of micrometers to several tenths of a micrometer are spontaneously formed when an excess volume of aqueous buffer is added to the dry lipid and the flask is agitated.

5. The "dry" lipid mixture is then hydrated in anaqueous medium containing buffers, salts, chelating agents, and the drug to be entrapped [98].

Preparation of small unilamellar liposomes: Highenergy sonic fragmentation processes were introduced in the early 1960s [100]. Modifications of these procedures using a high-pressure homogenization device followed [101]. SUVs are prepared by the following methodology to dissolve phospholipids in water to form optically clear suspensions.

\section{Sonication}

Methods for the preparation of sonicated SUVs have been reviewed in detail by Banghamet al [74]. Classically the MLV dispersion is placed in test tubes and sonicated either in a bath sonicator or by tip sonication. Normally a 5-10-min sonication procedure (above Tc) is sufficient to prepare SUVs with radii < $50 \mathrm{~nm}$. With some lipids, radii $<20 \mathrm{~nm}$ are also possible while some diacyl cationic lipids (including 1-[2-(oleoyloxy)-ethyl2-oleoyl-3-(2-hydroxyethyl) imidazolinium chloride (DOIC) and dioctadecylamidoglycylspermine (DOGS) can even form micelles. Dioctadecyldiammonium bromide (DOBAD) neutral lipid liposomes cannot be sized $<130 \mathrm{~nm}$ [101-102].

\section{Extrusion}

Pre-filtering the LMV solution through a filter with pores $\sim 1 \mu \mathrm{m}$ is followed by pre-filtering the solution five times through $0.4^{-}$and $0.2-\mu \mathrm{m}$ pores. This is followed by $5-10$ extrusions through a filter with a pore size of $100 \mathrm{~nm}$.Allowing the formation of LUVs with diameters slightly above presizes $(\sim 110-120 \mathrm{~nm})[98,102]$. If smaller vesicles are desired, continued filtering through $80-$ and $50-\mathrm{nm}$ pores is needed. Extrusion through smaller pores (30nm) or in the case of some more rigid bilayers, $50 \mathrm{~nm}$, does not reduce the size further but rather increases it owing to the imposition of too high a curvature to vesicles. The extrusion method yields the best vesicles with respect to the homogeneityof size distribution and to control the size distribution of vesicles, especiallyfor larger $(100-500 \mathrm{~nm})$ diameters [102].

Preparation of Large Uni-lamellar Liposomes: Large unilamellar liposomes denote to vesicles $>100 \mathrm{~nm}$ in diameter restricted to a single bilayer membrane. LUVs provide's a number of benefits compared to MLVs, including high encapsulation of water-soluble drugs, economy of lipid, and reproducible drug release rates [101]. These liposome's are the most difficult type of liposomes to produce; however, a number of techniques for producing LUVs such as freeze-thaw cycling, slow swelling in non-electrolytes, dehydration followed by rehydration, and the dilution or dialysis of lipids have been reported. The two primary methods used are one involving detergent dialysis, while the other uses the formation of a water-in-oil emulsion [101]. Detergents commonly used for this purpose exhibit a relatively high critical micelle concentration (CMC) such as bile salts and octylglucoside. During dialysis, when the detergent is removed, the micelles become progressively richer in phospholipid levels and finally coalesce to form closed, singlebilayer vesicles [101-102]. Liposomes (100 $\mathrm{nm}$ in diameter) are formed within a few hours.Uniform single-layered phosphor lipid vesicles of $100 \mathrm{~nm}$ are formed when sonicated, small phospholipid vesicles or dry phospholipid films are mixed with deoxycholate at a molar ratio of 1:2. Subsequently, the detergent is removed by passing over a Sephadex G-25 column [103]. This procedure separates $100-\mathrm{nm}$ vesicles from small sonicated vesicles [103]. The phospholipid solution is layered onto a sucrose gradient and subjected to high-speed centrifugation. The SUVs form as a sediment, leaving behind detergentin the supernatant layer. This procedure involves the removal of a nonionic detergent, Triton $\mathrm{X}-100$, from detergent/phospholipid miceller suspensions. Bio-Beads SM-2 have the ability to absorb Triton X-100 rapidly and selectively. Following absorption of the detergent, the beads are removed by filtration. The final liposome size depends 
on the conditions used including lipid composition, buffer composition, temperature, and, most importantly, the amount and the efficacy of the detergent-binding capacity of the beads. Additional procedure to prepare LUVs employs waterin-oil emulsions of phospholipids and buffer in excess. This method is principally useful to encapsulate a large amount of a water-soluble drug [103]. Two phases are usually emulsified by sonication. Removal of the organic solvent under the vacuum causes the phospholipid-coated droplets to coalesce and ultimately form a viscous gel. The removal of the final traces of solvent under a high vacuum or mechanical disruption results in the collapse of the gel into a smooth suspension of LUVs. To prepare reverse phase evaporation vesicle (REV)type liposomes, the phospholipids are first dissolved in either diethyl ether isopropyl ether or mixtures of two solvents such as isopropyl ether and chloroform. Emulsification is most easily accomplished if the density of the organic phase is $\sim 1$. The aqueous phase containing the material to be entrapped is added directly to the phospholipid-solvent mixture, forming a two-phase system. The ratio of aqueous phase to organic phase is maintained as 1:3 for ether and 1:6 for isopropyl etherchloroform mixtures. The two phases are sonicated for a few minutes, forming a water-in-oil emulsion, and the organic phase is carefully removed on a rotary evaporator at $20-30^{\circ} \mathrm{C}$. The removal of the last traces ofsolvent transforms the gel into large unilamellar liposomes [103-104] (Table 3).

\section{Preparation of ferrous sulfate liposomes}

Preparation of empty liposomes: Preparation of empty PCcholesterol liposomes: The empty PC-cholesterol liposomes were prepared by reverse-phase evaporation method (REV) [104]. The lipid mixture, containing egg PC (20mmol L_1) and various quantities of cholesterol (from 0 to $50 \mathrm{~mol} \%$ ), was dissolved in $30 \mathrm{~mL}$ diethyl ether. One-third volume (10 $\mathrm{mL}$ ) of buffer solution composed of $10 \mathrm{mmol}$ citric acid and $10 \mathrm{mmol} \mathrm{Na}_{2} \mathrm{HPO}_{4}$ (pH being adjusted to 6.8 ) was added to the organicphase [104]. Ultra-sonication with a probe sonicator (VCX400, Sonics \& Material Vibra Cell, $400 \mathrm{~W}, 20 \mathrm{kHz}$ ) in an ice bath for 5 min resulted in a homogeneous w/o emulsion. After the removal of the organic solvent at controlled reduced pressure with a rotary evaporator, a gel was formed. Upon continued rotary evaporation the gel was broken, and then the remaining aqueous phase $(20 \mathrm{~mL})$ was added with gentle vortexing. The remaining ether was evacuated at $0.01 \mathrm{MPa}$. The liposomes were stored at $4^{\circ} \mathrm{C}$ in a refrigerator [104].

\begin{tabular}{|c|c|c|c|}
\hline Vesicle Types & Abbrev & Diameter Size & Number of lipid bilayers \\
\hline $\begin{array}{l}\text { Small unilamellar } \\
\text { vesicles }\end{array}$ & SUV & $\begin{array}{l}\text { Diameter of } 20- \\
100 \mathrm{~nm} .\end{array}$ & One lipid bilayer \\
\hline $\begin{array}{l}\text { Large unilamellar } \\
\quad \text { vesicles }\end{array}$ & LUV & $\begin{array}{l}\text { Diameter of } \\
>100 \mathrm{~nm} \text {. }\end{array}$ & One lipid bilayer \\
\hline Multilamellar vesicles & MLV & Diameter of $>0.5 \mu \mathrm{m}$. & Five to twenty lipid bilayers \\
\hline Oligolamellar vesicles & OLV & $\begin{array}{l}\text { Diameter of } 0.1- \\
1 \mu \mathrm{m} .\end{array}$ & $\begin{array}{c}\text { Approximately five lipid } \\
\text { bilayers }\end{array}$ \\
\hline Multivesicular vesicles & MMV & Diameter of $>1 \mu \mathrm{m}$. & Multicompartmental \\
\hline
\end{tabular}

Preparation of empty Tween-grafted liposomes: Theempty Tween-grafted liposomes were also prepared byREV method. The lipid mixture contained egg PC (20mmol L_1) and cholesterol (2 mmol L_1). When thegel was broken, the remaining aqueous phase containing various quantities of Tween 80 surfactants (molar ratiofrom 0 to 1.0) was added. The other procedures were thesame as the above [104-105].

Preparation of ferrous sulfate liposomes: Based on the study of empty liposomes, the basic composition of the membrane fraction of the liposomes was fixed at $80 \%(\mathrm{~mol} /$ mol) egg lecithin, $10 \%(\mathrm{~mol} / \mathrm{mol})$ cholesterol and $10 \%(\mathrm{~mol} /$ mol) Tween 80. Ascorbic acid was used as an anti-oxidant to protect the ferrous ion against oxidation. Four kinds of methods were used to prepare ferrous sulfate liposomes in order to compare their encapsulation efficiency (EE) [104-105]. The most suitable method was selected according to the EE. Then parameters such as weight ratio of iron to lipids, hydrating media and sonication strength were optimized. The effect of weight ratio of iron to lipids (0.04, 0.06 and 0.1) on the EE was investigated. The effect of hydrating media on the EE was studied by encapsulating the ferrous sulfate solution (weight ratio of iron to lipids was 0.04) with different hydrating media (deionized water, $0.01 \mathrm{~mol} \mathrm{~L} \_1$ citric acid- $\mathrm{Na}_{2} \mathrm{HPO}_{4}$ buffer solution and $0.01 \mathrm{~mol} \mathrm{~L}$ _1citric acid-sodium citrate buffer solution). In addition, the effect of sonication strength $(60 \%$, $70 \%, 80 \%$ and $90 \%$ ) on the EE was studied [105].

Reverse-phase evaporation (REV): Ferrous sulfate liposome's were prepared by Fukuhi $\mathrm{H}$ et al [104] slightly differently from empty liposomes. In this system, one-third volume of aqueous solution containing ferrous sulfate and ascorbic acid (15:1 weight ratio of ferrous sulfate to ascorbic acid) was added to the organic phase. Final lipid concentration was about $30 \mathrm{mg} \mathrm{mL}$-1. Thinfilm hydration (TF) [104]: Lipids, at the above-indicated ratios, were dissolved in diethyl ether, in the adequate proportions, to obtain a limpid solution. Soon, the solvent was evaporated from the lipid solution in a rotary evaporator at controlled reduced pressure until a fine film was formed on the walls of the receptacle. It was compulsory to establish that no residue of the organic solvent remained, for that reason the lipid samples were placed under a high vacuum for at least $4 \mathrm{~h}$ to remove any residual solvent. Ferrous sulfate solution, containing Tween 80 , was introduced into the aforementioned receptacle and stirred up vigorously at ambient temperature. The agitation was continuous until there was no lecithin deposited on the wall, to give a final lipid concentration of approximately $30 \mathrm{mg} \mathrm{mL}-1$. Thin-film and sonication (TFS) [106]. After the thin-film hydration, the liposomes suspension was submitted to a probing sonication (Sonics \& Material Vibra Cell, $400 \mathrm{~W}, 20 \mathrm{kHz}$ ) process at $40 \%$ (sonication strength) in an ice bath for $5 \mathrm{~min}$ with a sequence of 1 s of sonication and 1s rest. The liposomes were stored at 40C. Freeze-thawing (FT) [105]. When the thin-film sonication liposome suspension had been obtained, it was rapidly frozen in a freezer at $180 \mathrm{oC}$ and left overnight. The next day, the suspension was spontaneously thawed at ambient temperature. The liposome suspension was subjected to three freeze-and-thaw cycles. The final liposomes were stored at $40 \mathrm{C}$. 
Preparation of biotin-peliposomes: Large unilamellar liposomes (LUVs; egg phosphatidylcholine [EPC]; Avanti Polar Lipids, Alabama) containing biotin-PE ( $0.1 \mathrm{~mol} \%$; Molecular Probes. Oregon, or Avanti Polar Lipids. Alabama), are prepared by extrusion techniques as described by Hope et al [106]. Concisely, appropriate amounts of lipid mixtures dissolved in chloroform are deposited in a tube and dried to a lipid film under a stream of nitrogen followed by high vacuum for $2 \mathrm{~h}$. Lipid samples are routinely hydrated in $150 \mathrm{mM} \mathrm{NaCl} .25 \mathrm{mM}$ $\mathrm{N}$-(2-hydroxyethyl) piperazine-N-3-propanesulfonic acid (EPPS), pH 8. Fortargeting experiments, a fluorescent marker such as carboxyfluoresceis included in the hydration buffer (15 $\mathrm{mM})$. The resulting multi-lamellar vesicles are frozen and thawed 5 times and extruded 10 times through 2 stacked 100nm filters [107].

Binding of strptavidin to biotin-Pe-Liposomes: Liposomes (99.9 mol\% EPC, 0.1 mol\% biotin-PE; 1 pmol total lipid) are normally incubated with streptavidin ( 1 l.KiWmg, $4 \mathrm{mg} / \mathrm{ml}$ in 20 mM EPPS. $150 \mathrm{mM} \mathrm{NaCl} \mathrm{pH} \mathrm{8.0;} \mathrm{Sigma} \mathrm{Chemical} \mathrm{Co.)} \mathrm{at} \mathrm{room}$ temperature for $30 \mathrm{~mm}$. Samples are then chromatographed on Sepharose CL-4B (Pharmacia. Uppsala. Sweden) which is equilibrated with EPPS.pH 8.0 to separate liposomal bound streptavidin from free. The ratio of streptavidin bound,lipid is determined by counting ' $=\mathrm{I}$ for streptavidin and a standard phosphate assay for phospholipid. Under the above conditions, a maximum of $5.8 \mathrm{~kg}$ of streptavidin binds per pmole of lipid [108]. The amount of streptavidin associated with vesicles is increased further by increasing the mol\% of biotin $\mathrm{PE}$ in the vesicles (up to $0.3 \mathrm{~mol} \%$ of the total lipid) or by the inclusion of cholesterol (Chol) in the vesicle preparation (50 mol\%). To maximize the coupling efficiency, a constant ratio of streptavidin to total lipid is maintained. Streptavidinliposomes with up to $30 \mathrm{u}, \mathrm{g}$ of protein bound per mol of lipid can readily be achieved in this manner. Further increases in the levels of biotin-PE in liposomes lead to a significant loss of lipid due to aggregation and precipitation of vesicles. Under the optimal incubation conditions outlined above. Protein to lipid coupling is rather inefficient. Only $3 \%$ of the initial protein becomes lipid associated. For this reasonthe rapid interaction of streptavidin with biotinated components may be more efficiently exploited by indirect targeting procedures. Whereby cells are sequentially labeled with a biotinated ligand, streptavidin and finally, biotin-PE liposomes [106-108].

Liposomal delivery of other drugs: The most active drugs against breast cancer are currently the anthracyclines and taxanes (paclitaxel and docetaxel). Schemes for the delivery of taxanes are under active research to increase tumor exposure and/or to reduce adverse effects such as neurotoxicity, edema, asthenia, and alopecia. In addition, special issues with the taxanes provide further rationale for the application of delivery systems [109]. Both paclitaxel and docetaxel are poorly soluble in aqueous solutions, and have consequently been formulated with vehicles Cremaphor EL and polysorbate 80 (TWEEN), respectively. These formulations are highly allergenic, require extensive premedication, and are responsible for most of the acute toxicities observed with taxane therapy, rather than the taxanes themselves. Delivery strategies in clinical trials include liposome-encapsulated paclitaxel] and poly(L-glutamic acid)paclitaxel, a polymer conjugate [109-110] (Figure 3).

Liposome-based drug delivery in breast cancer treatment: Liposomal anthracyclines have achieved highly effective drug encapsulation, resulting in substantial anticancer activity with reduced cardio toxicity, and include versions with greatly prolonged circulation such as liposomal daunorubicin and pegylated liposomal doxorubicin [109]. Pegylated liposomal doxorubucin has shown substantial efficacy in breast cancer treatment both as monotherapy and in combination with other chemotherapeutics. Additional liposome constructs are being developed for the delivery of other drugs. The next generation of delivery systems which include true molecular targeting; immune liposomes and other ligand-directed constructs represent an integration of biological components capable of tumor recognition with delivery technologies [109-110].

Delivery of anthracyclines: Anthracyclinesdemonstrates the case of potent anticancer activity that is guarded by highly problematic systemic toxicities. The most studied drug deliveryapplications in oncology have involved anthracyclines to reserve or to enhanceefficacy against tumor cells while limiting exposure to critical target sites such as myocardium and bone marrow.Anthracyclines have been encapsulated in a number of liposomal constructs [109]. Current varietiesexploit ion trapping methods to achieve extremely efficient loading of doxorubicin or daunorubicin within the aqueous interior of unilamellar (single bilayer) liposomes, reaching 104 drug molecules per liposome particle. Liposome-encapsulated doxorubicin (TLC D-99, Myocet ${ }^{\mathrm{TM}}$; Elan Pharmaceuticals, Inc., Cedar Knolls, NJ, USA) have developed a multiple vial kit, with discrete vials containing moderately sized $(\sim 190 \mathrm{~nm})$

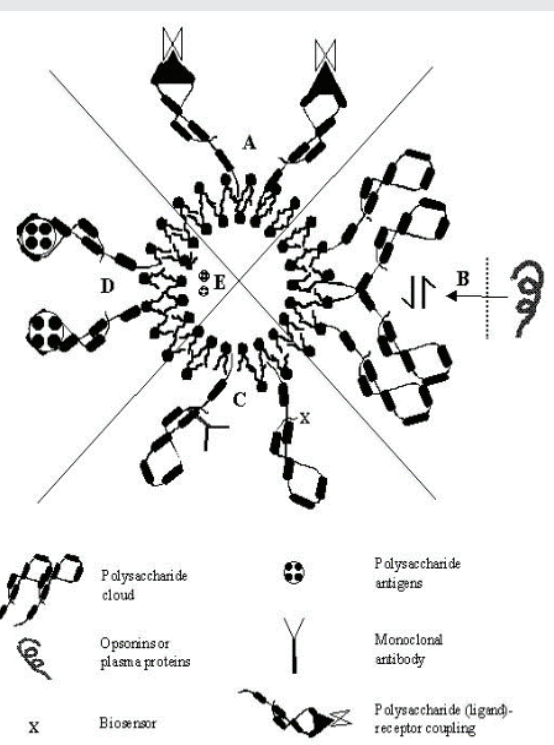

Figure 3: Various drug delivery, targeting and biotechnological areas where polysaccharide -bearing liposomes could be exploited as tools for future therapy. (A) Macrophage targeting based upon the ligand-receptor interaction. (B) Stealth behavior of the polysaccharide cloud, which further provides a hydrophilic environment to deter them from the opsonins and plasma proteins. (C) Polysaccharides as bio-sensors (targeting ligands) and offering bio-protection. (D) Vaccination potential of anchored bacterial polysaccharides or lipopolysaccharides. (E) Encapsulating polysaccharide antigens for vaccination [109]. 
liposomes, lyophilized doxorubicin, and citric acid buffer. The components are mixed at a point of care, resulting in highly efficient (>99\%) loading of doxorubicin into the liposomes. Myocet ${ }^{\mathrm{TM}}$ provides a limited degree of prolonged circulation as likened with free drug. However, the liposome encapsulation significantly alters the bio-distribution of doxorubicin, resulting in some reduction in toxicities [110].

Application's for delivery systems in breast cancer: Additional application's for delivery systems in breast cancer include approved chemotherapy drug's such as vinca alkaloid's, platinum's and camptothecin's. In each case, it's possible that delivery systems such as liposomes or polymers could improve pharmacokinetics, could increase tumor accumulation, and/ or could reduce limiting toxicities. Polymers capable of recognizing certain compounds by using the technique of molecular imprinting, which is more often associated with chromatography. The molecule that the polymer will sense is used as a template around which the monomers are allowed to polymerize. The template molecule is then extracted from the polymer [110-111]

Potential of polysaccharide anchored liposomes in drug delivery, targeting and immunization: Recently the prominence has been laid upon the carbohydrate mediated liposomal interactions with the target cells. Among the various carbohydrate ligands, such as glycoproteins, glycolipids, viral proteins, polysaccharides, lipo-polysaccharides and other oligosaccharides, this section deals with the polysaccharide anchored liposomal system for their potential in drug delivery, targeting and immunization [112-113]. Over the years, various schemes have been developed which include coating of the liposomal surface with natural or hydrophobized polysaccharides, namely manna, pullulan, amylopectin, dextran etc., or their palmitoyl or cholesteroyl derivatives. The polysaccharide(s) coat tends vesicular constructs physicochemical stable in bio-environments and site-specific. The aim of improving the physical and biochemical stability of liposomes and the ability to target liposomes to specific organs and cells, were the major attributes of the polysaccharide anchored liposomes [111-113] (Figure 4).

Polysaccharide Anchored Liposomes: In the development of polysaccharide anchored liposomes for therapeutic purposes, it is important to consider the mechanisms and methodologies of the polysaccharide link with the bilayer membrane and resultant effect on the bilayer permeability, fluidity, and integrity [114]. The affinity and the selectivity of the anchored polysaccharide towards it's paired ligand's is a desirable pre-requisite that makes the system site specific and target oriented. The impact of carbohydrate and polysaccharide specific recognition domains on the cell surface has stimulated the research quantitatively towards exploitation of technology to develop systems for drug's and/or antigen's [111-113]. Since the potential of natural or hydrophobized polysaccharides, methods have been developed to link polysaccharides to the surface of liposomes [106]. Earlier methods were attempted to anchor polysaccharides on the surface of the liposomes through adsorption, however recently spacer activated covalent coupling or hydrophobic anchoring have been valued as

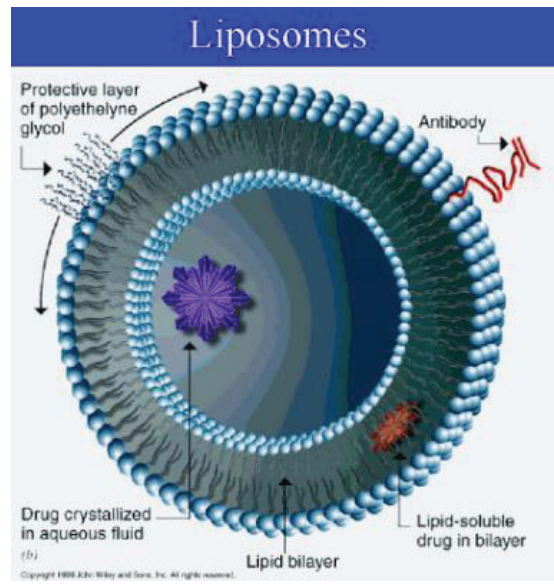

Figure 4: Liposomes used in cosmetic industry [112]

methods of anchoring. Earlier methods of anchoring exploited possible interaction of liposomes and polysaccharides. Sunamoto at. al., [106-107] investigated communications of simple polysaccharides and liposomal membranes and revealed that simple and naturally occurring polysaccharides, such as dextran, chitosan, pullulan, mannan, or amylopectin, powerfully adhere on to the liposomal surface mostly via hydrophobic interactions inducing subsequent aggregation and fusion of liposomes. Under specific conditions however, which do not allow for aggregation or fusion, the adsorption of polysaccharides over liposomal membranes may be due to diffusion controlled mechanism of constitutive components and coat, followed by lateral diffusion and subsequent interdigitization of adsorbed polysaccharide molecules into bilayers. This theory was later confirmed and substantiated by fluorescence depolarization technique using FITC-dextran as marker probe [106].

Polysaccharide anchoring by adsorption was found to be thermodynamically unstable and pharmaceutically unacceptable due to the following reasons:

1. The polysaccharides adsorbed on the liposomal surfaces easily desorb/delodge on dilution or on mechanical agitation.

2. Peptization or coagulation of the polysaccharides could lead to successive destabilization of the liposomal bilayer

3. Stoichiometric ligand density is often non-reproducible.

In order to prevent adsorptive coating related limitations, Sunamoto and Iwamoto [107] employed chemically modified polysaccharides, i.e., palmitoylated polysaccharides, to coat the liposomes. These partially hydrophobized polysaccharides were allowed to react covalently and subsequently integrate with the lipid constituents of liposomal membranes.

The surface modification of liposomes is mediated through the hydrophobic legs. Coating of liposomes with these hydrophobized polysaccharides can be achieved by incubation of aqueous solutions of polysaccharide derivative's with preformed liposomal dispersion. In some cases, Chol substituted 
polysaccharide was used to conjugate sensory devices like salicylic acid derivative or an IgM fragment [108]. The salicylic acid conjugated cholesterol substituted polysaccharide or immuno-polysaccharide derivatives were subsequently anchored over liposomes by dispersion-incubation technique under optimized standard conditions. The method have been used in the preparation of newly designed immune liposomes where PC based large oligolamellar vesicles were anchored to the polysaccharide pullulan. The system has been modified to carry both, i.e., cholesterol as a hydrophobic anchor, and monoclonal antibody fragment (anti-sialosyl Lewis x IgMs) as a sensory device. Various studies moreover reflect that polysaccharides anchored on liposomes using above-mentioned methods retained their ligand affinity and specificity [108].

\section{Therapeutic and clinical applications}

Polysaccharides have been developed in recent years for various delivery and targeting strategies, either they provided stabilization and formed a skeleton on which suitable sensory molecules were detached or otherwise they themselves behave as sensory devices to bring out the resultant targeted therapeutic effects. Some of the therapeutic benefits that polysaccharide anchored vesicles offer is well discussed [108].

Lung Therapeutics: Increased lung accumulation of polysaccharides anchored liposome promises for their selectivity and potential as drug delivery system for the therapy of lung diseases [109]. The alveolar macrophages selectively sequester the $\mathrm{O}$-palmitoyl amylopectin anchored liposomes. The macrophage cuptake have been confirmed with the help of fluorescent probe marker, as marker was traced mainly in macrophages after IV injection [110]. Moreover, OPA anchored liposomes are reported to be sequestered and retained selectively in lungs by anionic-scavenging receptors [110111]. Liposomes appended with O-palmitoylpullulan (OPP) and $O$-palmitoyl amylopectin (OPA) are swiftly cleared from the blood as compared to 'naked' liposomes. However, they have a relatively wide tissue circulation including liver and spleen, it's found that OPA anchored liposomes are selectively intercepted sequestered and internalized by the lung macrophages and monocytes. Subsequent to this observation, investigations were made on OPA anchored liposomes to explore their potential as a delivery system for sisomycin treatment of lung diseases in guinea pigs infected with Legionella pneumophila [110]. The therapeutically beneficial results of these studies subsequently promoted further investigations, where OPA anchored liposomes were tested for targeted delivery of antimicrobial agents against intracytoplasmic pathogens and fungus. Specifically, amylopectin anchored liposomes were found to be effective for the delivery of Ceftazidine to L Pneumophila infected guinea pigs where relative to treatment with free drug the survival rate achieved following the liposome treatment was $30 \%$.The liposomal encapsulated drug accumulation in the lung was two-fold higher compared to lung drug concentration following free drug administration. Miyazaki and coworkers [111] revealed that coating liposomes with amylopectin negotiates targeting of the incorporated amphotericin $\mathrm{B}$ to the lungs. The LD50 of amylopectin-anchored liposomal amphotericin B in normal mice was more than $10.0 \mathrm{mg} / \mathrm{kg}$, whilst for conventional amphotericin B, LD50 recorded to be $1.2 \mathrm{mg} / \mathrm{kg}$. Amylopectin-anchored liposomes showed two-fold higher accumulation in the lungs as compared to conventional liposomes. These workers further studied in vivo efficacy of the system using murine model of pulmonary candidiasis. Candida albicans was inoculated into BALB/C mice and the number of Candida in the lungs of mice treated with amylopectin-anchored liposomes and conventional liposomes were compared. The amylopectin-anchored liposomes improved the survival rate of inoculated mice [111].

Nano pores: Nanopores or micropores that optimistically remember only the specific template.When a solution of glucose, sucrose, and galactose, this particular compound would recognize only the glucose. In the example of glucosesensing molecularly imprinted nanoparticles, the detection of glucose would trigger the release of insulin from within the particle. As drugs become larger and less water soluble, the importance of new delivery systems will only increase [112].

Nano-liposomes and Their Applications in Food Nanotechnology: Food nanotechnology involves the exploitation of nano-carrier system's to stabilize the bio-active materials against a wide range of environmental and chemical changes as well as to improve their bio-availability [112]. Nano-liposome technology presents exciting opportunities for food technologists in areas such as encapsulation and controlled release of food materials, as well as the enhanced bio-availability, stability, and shelf-life of sensitive ingredients. Liposomes and nanoliposomes have been used in the food industry to deliver flavor's and nutrients and more recently, have been investigated for their ability to integrate antimicrobial's that could aid in the protection of food product's against microbial contamination [112]. The main physic-chemical properties of liposomes and nano-liposomes are industrially applicable methods for their manufacture are reviewed. There are quite huge applications of nano-liposomes as carrier vehicles of nutrients, enzymes, food additives, and food antimicrobials.

The Preparation and Testing of Liposomes and its Applications in Cosmetics: Liposome is one of the emerging in pharmaceutical and cosmetics in recent years.Existence of biological carrier,liposome loaded with active ingredients is very effective in treating disease and skin care. The result of Freeze fracture electromicrography showed the presence of liposomes.In the forgone conclusion that liposomes had good stability by measuring their particle size and microscopic structureand M. R. Muzafarriet. al., [111] also tested the encapsulation rate to the water soluble substance and discussed the application of liposomes in cosmetics as well. Liposomal formulations are the first Nano-Pharmaceuticals introduced to market, Doxil ${ }^{\circledR}$ PEGylated liposomal formulation for doxorubicin is the first product based on liposomes. These liposomes are called as "Stealth" liposomes with size $<200 \mathrm{~nm}$ which are long circulation with hydrophilic (PEG) surface. These long circulating liposomes found to target to tumour tissue by a mechanism known as enhanced permeation and retention (EPR). Hence liposomal formulation of doxorubicin considerably reduced the cardio-toxicity of drug [112-113]. 
Novel Nano-liposomal CPT-11 Infused by ConvectionEnhanced Delivery in Intracranial Tumors: Pharmacology and Efficacy: J. W. Park et. al., [112] postulated that combining convection-enhanced delivery (CED) with a novel, highly stable nanoparticle/liposome containing CPT-11 (nanoliposomal CPT-11) would provide a dual drug delivery strategy for brain tumor treatment. Following CED in rat brains, tissue retention of nano-liposomal CPT-11 was greatly prolonged, with $>20 \%$ injected dose remaining at 12 days for all doses. Tissue residence was dose dependent, with doses of $60 \mathrm{\mu g}$ (3 $\mathrm{mg} / \mathrm{mL}), 0.8 \mathrm{mg}(40 \mathrm{mg} / \mathrm{mL})$, and $1.6 \mathrm{mg}(80 \mathrm{mg} / \mathrm{mL})$ resulting in tissue half-life (t1/2) of $6.7,10.7$, and 19.7 days, respectively [113]. In contrast, CED of free CPT-11resulted in rapid drug clearance (tissue $\mathrm{t} 1 / 2=0.3$ day). At equivalent CED doses, nano-liposomal CPT-11 increased area under the timeconcentration curve by 25 -fold and tissue $\mathrm{t} 1 / 2$ by 22 -fold over free CPT-11; CED in intracranial U87 gliomaxenografts showed even longer tumor retention (tissue $\mathrm{t} 1 / 2=43$ days). Plasma levels were undetectable following CED of nano-liposomal CPT-11. Importantly, prolonged exposure to nano-liposomal $\mathrm{CPT}-11$ resulted in no measurable central nervous system (CNS) toxicity at any dose tested (0.06-1.6 mg/rat), whereas CED of free CPT-11 induced severe CNS toxicity at $0.4 \mathrm{mg} / \mathrm{rat}$. In the intracranial U87 gliomaxenograft model, a single CED infusion of nano-liposomal CPT-11 at $1.6 \mathrm{mg}$ resulted in significantly improved median survival ( $>100$ days) compared with CED of control liposomes (19.5 days; $\mathrm{P}=4.9 \times 10-5)$ or free drug $(28.5$ days; $\mathrm{P}=0.011)$. The CED of nano-liposomal CPT-11 greatly prolonged tissue residence while also substantially reducing toxicity, resulting in a highly effective treatment strategy in preclinical brain tumor models [113].

Nano-liposome Delivers Anticancer Drug to Brain Tumors, Avoids Healthy Tissue: Uniqueit is why patients with brain cancer face such a poor diagnosis is that there are so fewanticancer drugs that can actually cross the so-called blood-brain barrier and reach tumors growing in the brain [114]. But using a nano-scale, drug-loaded liposome and a pressure-driven drug administration technique known as convection-enhanced delivery, investigators at the University of California at San Francisco (UCSF) have developed an efficient method of getting anticancer drugs into the brain and keeping them there. This new approach can also reduce the therapy-limiting toxicities that are often associated with the most potent anticancer agents [114] (Figure 5).

Loading nanoliposome therapeutics in red blood cells using electroporation: Amrita Mehta et.al., [114] reported prepared a novel drug delivery system by loading nanoliposome therapeuticsin human red blood cells (RBCs) by electroporation, and to enhance drugcirculationprofilesin vivo. During the studies conducted by A. Mehta et. Al.,[114] rigorous evaluation of delivery approach, A.Mehta et al [114], have determined that these specific type andconcentration of liposomes, as well as the number of electroporation pulses used are importantconsiderations for efficient loading of nano-systems in RBCs. They [114] alsoinvestigated therole of a cell preservative solution on the viability of electroporated red bloodcells, in an effort toincrease the life span of the cells following reinjection.

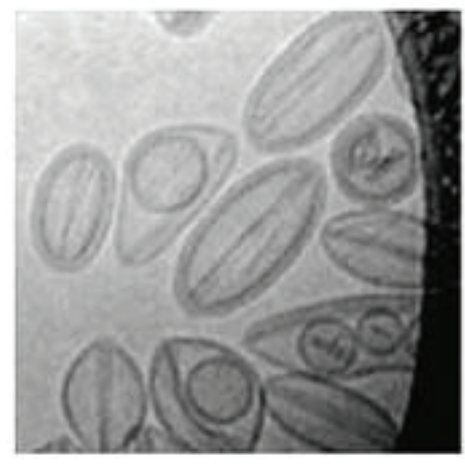

Figure 5: Nanoliposome Delivery May Improve Cancer Drug Activity [114].

Nano-liposome Delivery May Improve Cancer Drug Activity: The use of nano-carriers will allow the creation of formulations that are highly active against solid tumors and can be targeted to cell-surface receptors, the researchers said. "Our technology forms a remarkably stable complex between the drug and a high-charge-density polyanion in the liposomal lumen [115-116]. The stability of this complex helps govern the rate of release of the drug from the carrier, thus ensuring that it can localize to the tumor to a significant extent before it is released," said Daryl C. Drummond, PhD, senior director for liposomal discovery at Merrimack Pharmaceuticals in Cambridge [115-118].

\section{Conclusion}

Mini review articulates the significance of liposome drug delivery systems are designed tostable and efficiently carry anticancer agents to tumor sites. This has been accomplished with some success by liposomal versions of anthracyclines. Current liposomal agents, nanolipids, anthracyclines provide improved pharmacokinetics, provide reduced toxicities to a number of organ sites, and provide potentially increased tumor uptake. The next generation of delivery systems in development combine these features with tumor cell recognition, and include antibody-targeted and cellinternalizing systems. Such systems will enable drug delivery to move beyond pharmacokinetic-driven and bio-distributiondriven mechanisms to true molecular targeting. This trend can also be viewed as an integration of biological therapeutics and drug delivery technologies. Various methods of preparation of liposomes have been depicted which could give good activity for drug delivery systems, ex: nano liposomes or nanophopholipids. It is probable that such integrated approacheswill include biologically targeted delivery systems for small molecule drugs as well as for biological agents with anticancer activity, and will be an increasingly important theme in the development of new treatments of breast cancer, heart ailments, paralysis, AIDS and dreadful diseases which is the need of the hour.

\section{References}

1. D. Chapman (1983) Liposomal technology 1: 1-18.

2. HAH Rongen, A Bult, WP van Bennekom (1997) Liposomes and immunoassays. J Immuno Methods 204: 105-133. Link: https://tinyurl.com/y9kax4gx

3. DJA Crommelin (1978) Liposomes 3: 73-190 4194-4198. 
4. D Deamer, AD Bangham (1976) Large volume liposomes by an ether vaporization method Biochem Biophys Acta 443: 629-634. Link: https://tinyurl.com/y8rplmz2

5. Lipids For Liposome Formation. Link: https://tinyurl.com/yb383knn

6. Karmali PP, Chaudhuri A (2007) Cationic liposomes as non-viral carriers of gene medicines: resolved issues, open questions, and future promises. Med Res Rev 27: 696-722. Link: https://tinyurl.com/yaznt8x3

7. Deepthi V, Kavitha A (2014) Liposomal drug delivery system: a review. RGUHS J Pharm Sci 4: 47-56. Link: https://tinyurl.com/y9cj2uor

8. Lemie're J, Carvalho K, Sykes C (2015) Cell-sized liposomes that mimic cell motility and the cell cortex. Methods Cell Biol 128: 271-285. Link https://tinyurl.com/yazo3hns

9. Sercombe L, Veerati T, Moheimani F, Wu SY, Sood AK, et al. (2015) Advances and challenges of liposome assisted drug delivery. Front Pharmacol 6: 286 Link: https://tinyurl.com/ycqcoby5

10. Mouritsen O (2011) Lipids, curvature, and nano-medicine. Eur J Lipid Sci Technol 113: 1174-1187. Link: https://tinyurl.com/y7ddhajc

11. Sharma PH, Powar PV, Sharma SS (2014) Pharmacosomes: a novel drug delivery system. Pharma Innov J 3: 94-100. Link: https://tinyurl.com/y9vwtqry

12. Hua S, Wu SY (2013) The use of lipid-based nanocarriers for targeted pain therapies. Front Pharmacol 4: 143. Link: https://tinyurl.com/y9j8hdn2

13. Zhang LF, Granick S (2006) How to stabilize phospholipid liposomes (using nanoparticles). Nano Lett. 6: 694-698. Link: https://tinyurl.com/yd2nuoq5

14. Michel R, Plostica T, Abezgauz L, Danino D , Gradzielski M (2013) Control of the stability and structure of liposomes by means of nanoparticles. Soft Matter 9: 4167-4177. Link: https://tinyurl.com/yc968dqh

15. Liu JW, Jiang XM, Ashley C, Brinker CJ (2009) Electrostatically Mediated Liposome Fusion and Lipid Exchange with a Nanoparticle-Supported Bilayer for Control of Surface Charge, Drug Containment, and Delivery. J Am Chem Soc.131: 7567-7569. Link: https://tinyurl.com/y9kouojt

16. Liu JW, Stace-Naughton A, Jiang XM, Brinker CJ (2009) Porous Nanoparticle Supported Lipid Bilayers (Protocells) as Delivery Vehicles. J Am Chem Soc 131: 1354-1355. Link: https://tinyurl.com/ybn25gdl

17. Gao WW, Hu CMJ, Fang RH, Zhang LF (2013) Liposome-like nanostructures for drug delivery. J Mater Chem B. 1: 6569-6585. Link: https://tinyurl.com/ycmwq3ee

18. Batist G, Sawyer M, Gabrail N, N.Christiansen , J.L.Marshall, et al. (2008) A multicenter, phase II study of $\mathrm{CPX}-1$ liposome injection in patients (pts) with advanced colorectal cancer (CRC). J Clin Oncol 26: 4108. Link: https://tinyurl.com/y8e7nzto

19. Batist G, Sawyer M, Hart K, A Louie (2010) Phase 2 study of CPX-1liposome injection: UGT1A1 and prediction of severe toxicities. Ann Oncol 19: 103.20 Rodríguez-AntonaC Pharmacogenomics of paclitaxel. Pharmacogenomics 11: 621-623. Link: https://tinyurl.com/y75fygno

20. Minotti G, Menna P, Salvatorelli E, Cairo G , Gianni L (2004) Anthracyclines:molecular advances and pharmacologic developments in antitumor activity and cardiotoxicity. Pharmacol Rev 56:185-229. Link: https://tinyurl.com/ycre5u6t

21. Rahman AM, Yusuf SW, Ewer MS (2007) Anthracycline-induced cardiotoxicity and the cardiac-sparing effect of liposomalformulation. Int $\mathrm{J}$ Nanomedicine 2: 567-583. Link: https://tinyurl.com/yc7you6b

22. Barenholz Y. Doxil_ e (2012) the first FDA-approved nano-drug: lessons learned. J Control Release 160:117e134. Link: https://tinyurl.com/ybaf55gt
23. Gabizon A, Goren D, Fuks Z, et al (1983) Enhancement ofadriamycin delivery to liver metastatic cells with increased tumoricidal effect using liposomes as drug carriers. CancerRes 43:4730e4735. Link: https://tinyurl.com/y8t3fr9x

24. Hofheinz RD, Gnad-Vogt SU, Beyer U, et al (2005) Liposomalencapsulated anti-cancer drugs. Anticancer Drugs 16: 691 e707.

Link: https://tinyurl.com/yb8juj3y

25. Harris L, Batist G, Belt R, et al (2002) Liposome-encapsulated doxorubicin compared with conventional doxorubicin in arandomized multicenter trial as first-line therapy ofmetastatic breast carcinoma. Cancer 94:25e36. Link: https://tinyurl.com/yczofedr

26. O'Brien ME, Wigler N, Inbar M, et al (2004) Reduced cardiotoxicityand comparable efficacy in a phase III trial of pegylatedliposomal doxorubicin $\mathrm{HCl}$ (CAELYX/Doxil) versus conventional doxorubicin for first-line treatment ofmetastatic breast cancer. Ann Oncol 15:440e449. Link: https://tinyurl.com/y85evq7x

27. Carbone PP, Bono V, Frei E 3rd, Brindley CO (1963) Clinical studies with vincristine. Blood 21:640-647. Link: https://tinyurl.com/ycuclblg

28. Kantarjian HM, O'Brien S, Smith TL, et al (2000) Results of treatment with hyper-CVAD, a dose-intensive regimen, in adult acute lymphocytic leukemia J ClinOncol 18: 547-561. Link: https://tinyurl.com/ybmnprb4

29. Larson RA, Dodge RK, Burns CP, et al (1995) A five-drug remission induction regimen with intensive consolidation for adults with acute lymphoblastic leukemia: Cancer and Leukemia Group B study 8811. Blood 85: 2025-2037. Link: https://tinyurl.com/ycl8fhoq

30. Linker CA, Levitt LJ, O'Donnell M, Forman SJ, Ries CA (1991) Treatment of adult acute lymphoblastic leukemia with intensive cyclical chemotherapy: a follow-up report. Blood 78: 2814-2822. Link: https://tinyurl.com/y95aa5qq

31. Lamanna N, Heffner LT, Kalaycio M, et al (2013) Treatment of adults with acute lymphoblastic leukemia: do the specifics of the regimen matter? Results from a prospective randomized trial.Cancer 119: 1186-1194. Link: https://tinyurl.com/y9d5qwx6

32. Millar JL, Millar BC, Powles RL, et al (1998) Liposomal vincristine for the treatment of human acute lymphoblastic leukaemia in severe combined immuno deficient (SCID) mice. $\mathrm{Br} J$ Haematol 102: 718-721. Link: https://tinyurl.com/yczfvjn5

33. Owellen RJ, Hartke CA, Dickerson RM, Hains FO (1976) Inhibition of tubulinmicrotubule polymerization by drugs of the vinca alkaloid class.Cancer Res 36:1499-1502. Link: https://tinyurl.com/y8kajy2v

34. Sarris AH, Hagemeister F, Romaguera J, et al (2000) Liposomal vincristine in relapsed non-Hodgkin's lymphomas: early results of an ongoing phase II trial. Ann Oncol 11:69-72. Link: https://tinyurl.com/yc46fb38

35. Hoelzer D (1994) Treatment of acute lymphoblastic leukemia. SeminHemato 31:1-15.

36. Mayer LD, Nayar R, Thies RL, Boman NL, Cullis PR, Bally MB (1993) Identification of vesicle properties that enhance the antitumour activity of liposomal vincristine against murine L1210 leukemia.Cancer ChemotherPharmacol 33:17-24. Link: https://tinyurl.com/yag5gz5l

37. Boman NL, Bally MB, Cullis PR, Mayer LD, Webb MS (1995) Encapsulation of vincristine in liposomes reduces toxicity and improves its anti-tumor efficacy. J Liposome Res 5: 523-541. Link: https://tinyurl.com/y8xtyemh

38. Owellen RJ, Root MA, Hains FO (1977) Pharmacokinetics of vindesine and vincristine in humans.Cancer Res 37: 2603-2607. Link: https://tinyurl.com/yd338du

39. Sethi VS, Jackson DV Jr, White DR, et al (1981) Pharmacokinetics of vincristine sulfate in adult cancer patients.Cancer Res 41: 3551-3555. Link: https://tinyurl.com/yanq76wj 
40. Haim N, Epelbaum R, Ben-Shahar M, Yarnitsky D, Simri W, Robinson E (1994) Full dose vincristine (without 2-mg dose limit) in the treatment of lymphomas. Cancer 73: 2515-2519. Link: https://tinyurl.com/ybcrtrwh

41. Layton D, Trouet A (1980) A comparison of the therapeutic effects of free and liposomally encapsulated vincristine in leukemic mice.Eur J Cancer 16: 945-950. Link: https://tinyurl.com/y9v5nj8s

42. Mayer LD, Tai LC, Ko DS (1989) Influence of vesicle size, lipid composition, and drug-to-lipid ratio on the biological activity of liposomal doxorubicin in mice. Cancer Res 49: 5922-5930. Link: https://tinyurl.com/ybbd9zdg

43. Bally MB, Mayer LD, Loughrey H (1988) Dopamine accumulation in large unilamellar vesicle systems induced by transmembrane ion gradients. Chem Phys Lipids 47: 97-107. Link: https://tinyurl.com/yb8dugyc

44. Mayer LD, Wong KF, Menon K, Chong C, Harrigan PR, et al.(1988) Influence of ion gradients on the transbilayer distribution of dibucaine in large unilamellar vesicles. Biochemistry 27: 2053-2060. Link: https://tinyurl.com/yblsbb72

45. Mayer LD, Bally MB, Loughrey H, Masin D, Cullis PR (1990) Liposoma vincristine preparations which exhibit decreased drug toxicity and increased activity against murine L1210 and P388 tumors. Cancer Res 50: 575-579. Link: https://tinyurl.com/y9c932ny

46. Mayer LD, Nayar R, Thies RL, Boman NL, Cullis PR, et al. (1993) Identification of vesicle properties that enhance the antitumour activity of liposomal vincristine against murine L1210 leukemia. Cancer ChemotherPharmacol 33: 17-24. Link: https://tinyurl.com/y9pxh9wv

47. Krishna R, Webb MS, St Onge G, Mayer LD (2001) Liposomal and nonliposomal drug pharmacokinetics after administration of liposome-encapsulated vincristine and their contribution to drug tissue distribution properties. $J$ PharmacolExpTher 298: 1206-1212. Link: https://tinyurl.com/y9gywgf2

48. Webb MS, Harasym TO, Masin D, Bally MB, Mayer LD (1995) Sphingomyelincholesterol liposomes significantly enhance the pharmacokinetic and therapeutic properties of vincristine in murine and human tumour models. $\mathrm{Br}$ J Cancer 72: 896-904. Link: https://tinyurl.com/ycmeg8b8

49. Wong M, Kantarjian H, Appelbaum F (2007) Vincristine sulfate liposomes injection concentrates vincristine in tumor tissue and bone marrow of tumor bearing mice. American Association for Cancer Research - National Cancer Institute - European Organisation for Research and Treatment of Cancer: International Conference on Molecular Targets and Cancer Therapeutics 22 26. Link: https://tinyurl.com/y9dsufp3

50. Kanter PM, Klaich GM, Bullard GA, King JM, Bally MB, et al. (1994) Liposome encapsulated vincristine: preclinical toxicologic and pharmacologic comparison with free vincristine and empty liposomes in mice, rats and dogs Anticancer Drugs 5: 579-590. Link: https://tinyurl.com/yad97rdc

51. Leonetti C, Scarsella M, Semple SC (2004) In vivo administration of liposomal vincristine sensitizes drug-resistant human solid tumors. Int J Cancer 110 767-774. Link: https://tinyurl.com/y8ky9tcc

52. Silverman JA, Deitcher SR (2013) Marqibo ${ }^{\circledR}$ (vincristine sulfate liposome injection) improves the pharmacokinetics and pharmacodynamics of vincristine. Cancer Chemother Pharmacol 71: 555-564. Link: https://tinyurl.com/ych95qly

53. TagamiT, May JP, Ernsting MJ, Li SD (2012) A thermosensitive liposome prepared with a Cu2+ gradient demonstrates improved pharmacokinetics, drug delivery and antitumor efficacy. J Control Release 161: 142-149. Link: https://tinyurl.com/y863vrc4

54. Park SM, Kim MS, Park SJ (2013) Novel temperature-triggered liposome with high stability: formulation, in vitro evaluation, and in vivo study combined with high-intensity focused ultrasound (HIFU). J Control Release 170: 373-379. Link: https://tinyurl.com/y89aru7x

55. Chiu GN, Abraham SA, Ickenstein LM (2005) Encapsulation of doxorubicin into thermosensitive liposomes via complexation with the transition metal manganese. J Control Release 104: 271-288. Link: https://tinyurl.com/y7kxp2s9

56. Viglianti BL, Ponce AM, Michelich CR (2006) Chemodosimetry of in vivo tumor liposomal drug concentration using MRI. MagnReson Med 56: 1011-1018. Link: https://tinyurl.com/yalrvz6a

57. Nabholtz JM, Falkson C, Campos D, Szanto J, Martin M (2003) Docetaxel and doxorubicin compared with doxorubicin and cyclophosphamide asfirst-line chemotherapy for metastatic breast cancer: results of a randomized, multicenter, phase Illtrial. J ClinOncol 21 :968-975. Link: https://tinyurl.com/yct6bw6r

58. Tannock IF, de Wit R, Berry WR, Horti J, Pluzanska A (2004) Docetaxel plus prednisone or mitoxantroneplusprednisone for advanced prostate cancer. $\mathrm{N}$ Engl J Med 351 :1502-1512. Link: https://tinyurl.com/y9pev8w4

59. Fossella FV, DeVore R, Kerr RN, Crawford J, Natale RR (2000) Randomizedphase III trial of docetaxel versus vinorelbine or ifosfamide in patients with advanced non-smallcelllung cancer previously treated with platinum-containing chemotherapy regimens. The TAX 320Non-Small Cell Lung Cancer Study Group.J ClinOncol18: 2354-2362.

60. Fossella F, Pereira JR, von Pawel J, Pluzanska A, Gorbounova V (2003) Randomized, multinational, phase III study ofdocetaxel plus platinum combinations versus vinorelbine plus cisplatin for advanced non-smallcelllung cancer: the TAX 326 study group. J ClinOncol 21: 3016-3024. Link: https://tinyurl.com/y76m8t7c

61. Vermorken JB, Remenar E, van Herpen C, Gorlia T, Mesia R (2007) Cisplatin, fluorouracil, and docetaxel inunresectable head and neck cancer. $\mathrm{N}$ Engl J Med 357: 1695-1704. Link: https://tinyurl.com/y97btuw5

62. Posner MR, Hershock DM, Blajman CR, Mickiewicz E, Winquist E, et al. (2007) Cisplatin and fluorouracil alone or with docetaxel in head and neck cancer. N Engl J Med 357: 1705-1715. Link: https://tinyurl.com/y89l9qoq

63. Van Cutsem E, Moiseyenko VM, Tjulandin S, Majlis A, Constenla M, et al. (2006) Phase III study of docetaxel and cisplatin plusfluorouracil compared with cisplatin and fluorouracil as first-line therapy for advanced gastriccancer: a report of the V325 Study Group. J ClinOncol 24: 4991-4997. Link: https://tinyurl.com/ybmvc4ya

64. Taxotere package insert. Link: https://tinyurl.com/ychz2/4h

65. Sharpe M, Easthope SE, Keating GM, Lamb HM (2002) Polyethylene glycolliposomal doxorubicin: a review of its use in the management ofsolid and haematological malignancies and AIDS-related Kaposi's sarcoma. Drugs 62 2089-2126. Link: https://tinyurl.com/yaza2qyo

66. Park J (2002) Liposome-based drug delivery in breast cancer treatment. Breast Cancer Res 4: 95-99. Link: https://tinyurl.com/y7n3v75f

67. Schwonzen M, Kerbacher C, Mallmann P (2000) Liposomal doxorubicin and weekly paclitaxel in the treatment of metastatic breast cancer. Anticancer Drugs 11: 681-685. Link: https://tinyurl.com/y9brfyhb

68. Johnston S, Gore M (2001) Caelyx: phase II studies in ovarian cancer. Eur J Cancer 37(Suppl9): S8-S14. Link: https://tinyurl.com/y83lf6dp

69. Gordon AN, Fleagle JT, Guthrie D, Parkin DE, Gore ME, et al. (2001) Recurrent epithelial ovarian carcinoma: a randomized phase III study of pegylated liposomal doxorubicin versus topotecan. JClinOncol 9: 3312-3322. Link: https://tinyurl.com/yar9vbu5

70. Stewart S, Jablonowski H, Goebel FD, Arasteh K, Spittle M, et al. (1998) Randomized comparative trial of pegylated liposomal doxorubicin versus bleomycinand vincristine in the treatment of AIDS-related Kaposi's sarcoma. International Pegylated Liposomal Doxorubicin Study Group. J Clin Oncol 16: 683-691. Link: https://tinyurl.com/yct7qkdg 
71. J A Treat, C Huang, N Damjanov, S Walker, P Drobins, et al. (2000) Phase I tria in advanced malignancies with liposome encapsulated paclitaxel (LEP). Clin Cancer Res 6: 4492s. Link: https://tinyurl.com/ya57r4fn

72. Chapman CJ, Erdahl WE, Taylor RW, Pfeiffer DR (1991) Effects of solute concentration on the entrapment of solutes in phospholipid vesicles prepared by freeze-thaw extrusion. Chem Physic Lipid 60: 201-208. Link: https://tinyurl.com/y8epegkq

73. Bangham AD, Standish MM, Watkin JC (1965) Diffusion of univalent ions across the lamellae of swollen phospholipids. J Mol Biol 13: 238-252. Link https://tinyurl.com/yaqoju59

74. Kirby C, Gregoriadis G (1984) Dehydration-Rehydration Vesicles: A Simple Method for High Yield Drug Entrapment in Liposomes. Biotechnology 2: 979 984. Link: https://tinyurl.com/yaxvk9j2

75. Kimball's Biology Pages, “Cell Membranes.” Link: https://tinyurl.com/yb3ta78n

76. S Stryer (1981) Biochemistry. 213

77. New RR, Chance ML, Heath S (1981) Antileishmanial activity of amphotericin and other antifungal agents entrapped in liposomes. $J$ antimicrob Chemother 8: 371-381. Link: https://tinyurl.com/ydf2htb4

78. Taylor RL, Williams DM, Craven PC, Graybill JR, Drutz DJ, et al. (1982) Amphotericin B in liposomes: a novel therapy for histoplasmosis. Am Rev Respir Dis 125: 610-611. Link: https://tinyurl.com/ybp7xd4v

79. Graybill JR, Craven PC, Taylor RL, Williams DM, Magee WE (1982) Treatment of murine cryptococcosis with liposome-associated amphotericin B. J Infect Dis 145: 748-752. Link: https://tinyurl.com/yaw54kkw

80. Lopez-Berestein G, Mehta R, Hopfer RL, Mills K, Kasi L, et al. (1983) Treatment and prophylaxis of disseminated infection due to Candida albicans in mice with liposome-encapsulated amphotericin B. J Infect Dis 147: 939-945. Link: https://tinyurl.com/y8zml5e3

81. Lopez-Berestein G, Mehta R, Hopfer RL, Mills K, Kasi L, et al. (1983) Treatment and prophylaxis of disseminated infection due to Candida albicans in mice with liposome-encapsulated amphotericin B. J Infect Dis 147: 939-945. Link: https://tinyurl.com/y8zml5e3

82. Hopfer RL, Mills K, Mehta R, Lopez-Berestein G, Fainstein V, et al. (1984) In vitro antifungal activities of amphotericin $B$ and liposome-encapsulated amphotericin B. Antimicrobial Agents Chemorther 25: 387-389. Link: https://tinyurl.com/ydg7udby

83. Adler-Moore JP, Chiang SM, Satorius A, Guerra D, Andrews B, et al. (1984) J AntimicrobChemother; 28: 63-71.

84. Abra RM, Hunt CA, Lau DT (1984) Liposome disposition in vivo.VI: Delivery to the lung. J Pharm Sci 73: 203-206. Link: https://tinyurl.com/yccak2dm

85. SzokaJr FC, Milholland D, Barza M (1987) Effect of lipid composition and liposome size on toxicity and in vitro fungicidal activity of liposomeintercalated amphotericin B. Antimicrob Agents Chemother 31: 421-429. Link: https://tinyurl.com/y9bd2to3

86. Bangham AD, Standish MM, Watkin JC (1965) Diffusion of univalent ions across the lamellae of swollen phospholipids. J MolBiol 13: 238-252. Link https://tinyurl.com/yaqoju59

87. Gold JW (1984) Am J Med 76: 485-63.

88. Gilman, Rall TW, Nies AS, Taylor P (1985) (New York Macmillan), Sande MA, Mandell GL InThe Pharmacological Basis of Therapeutics. Editors AG. 12191222.

89. Clements JS, Peacok JE (1990) Amphotericin B revisited: reassessment of toxicity. J Med Am 88: 22N-7N. Link: https://tinyurl.com/yb8clpex

90. Meunier F, Prentic HG, Ringden 0 (1991) J Antimicrob Chemother 28: 83-91
91. Alvin CR, Sivartz GM., Jr. (1984) Liposome Techology, vol. II. CRC Press, Boca Raton, FL, 55-69.

92. Szoda FC, Papahadjopoulos D (1981) Liposomes: preparation and characterization, in Liposomes: From Physical Structure to Therapeutic Application Knight, C. G.ed. Elsevier 51-82. Link: https://tinyurl.com/y8oxe4va

93. Payne NL, Browning I, Haynes CA (1986) Characterization of Proliposomes. J. Pharmaceut. Sci. 75: 330-333. Link: https://tinyurl.com/ybxwazkw

94. Saunders L, Perrin J, Gammack DB (1962) Ultrasonic irradiation of some phospholipids sols. J. Pharmaceut. Pharmacol. 14: 567-572. Link: https://tinyurl.com/y9cqyne6

95. Huang $\mathrm{CH}$ (1969) Studies on phosphatidylcholine vesicles. Formation and physical characteristics. Biochemistry 8: 344-351. Link: https://tinyurl.com/ycqaa33r

96. Lasic DD (1993) Liposomes: from physics to application. Netherlands: Elsevier 575. Link: https://tinyurl.com/yadposnx

97. Paoli DT, Hager AA, Adelina V (1996) Liposomes containing bioavailable Iron (II) and process for obtaining them. US patent 5,534,268. Link: https://tinyurl.com/ydghjaa6

98. Basu S, Basu M, (2008) Liposome methods and protocols.199.

99. Laughrey L, Baily MB, Ctdlis PR (1987) preparation of streptavidin-liposomes for use in ligand-specific targeting applications. Biop@. Acra. 901. 157. Link: https://tinyurl.com/y8zuhv5e

100. Matsumura $Y$, Maeda H (1986) A new concept for macromolecular therapeutics in cancer chemotherapy: mechanism of tumoritropic accumulation of proteins and the antitumor agent smancs. Cancer Res 6 : 193-210. Link: https://tinyurl.com/y84a92fb

101. (2002) Liposome-based drug delivery in breast cancer treatment. UCSF Comprehensive Cancer Center, San Francisco, California, USA Breast Cancer Res 4: 95-99 Link: https://tinyurl.com/y873xa7o

102. (2001) Pharm PharmaceutSci (www.ualberta.ca/ csps) 4: 138-158.

103. Iwamoto K, Kato T, Kawahara M, Koyama N, Watanabe S, et all. (1991) Polysaccharide-anchored oil droplets in oil-in-water emulsions as targetable carriers for lipophilic drugs. J Pharm Sci 80: 219-224. Link: https://tinyurl.com/yc86c46b

104. Fukui H1, Akiyoshi K, Sunamoto J (1996) O/w-emulsion of alpha-linolenic acid stabilized with hydrophobized polysaccharide. Its effect on the growth of humancolon cancer cells. J BiomaterSciPolym Ed 7: 829-838. Link: https://tinyurl.com/yblrwgyf

105. Akiyoshi K, Kobayashi S, Shichibe S, Mix D, Baudys M, et all. (1998) Self-assembled hydrogel nanoparticle of cholesterol-bearing pullulanas a carrier of protein drugs: complexation and stabilization of insulin. $J$ Control Rel, 54: 313-320. Link: https://tinyurl.com/y93o9tuj

106. Sunamoto J, Sato T, Taguchi T, Hamazaki H (1992) Macromolecules, 25 : 5665-5670. Link: https://tinyurl.com/ycxfgh3s

107. Sunamoto J, Iwamoto K, Takada M, Yujuriha T, Katayama K (1984) Recent Advances in Drug Delivery Systems. Plenum Press, NY 153-162.

108. Takada M, Yuzuriha T, Katayama K, Iwamoto K, Sunamoto J (1984) Biochim BiophysActa, 802: 237-244,. Link: https://tinyurl.com/ybdll8zv

109. Sunamoto J, Iwamoto K, Takada M, Yuzuriha T, Katayama K (1983) Polym Sci Technol 23: 157-168.

110. Miyazaki T, Kohno S, Sasayama K, Inoue Y, Hara K, et all. (1992) Tohoku J Exp Med 168: 483-490. Link: https://tinyurl.com/y73jeczz

111. Mozafari MR, Johnson C, Hatziantoniou S, Demetzos C (2008) 
Nanoliposomes and Their Applications in Food Nanotechnology DOI 10.1080/08982100802465941. Link: https://tinyurl.com/y9ejjerl

112. John W Park, Kirpotin DB, Drummond DC, Yi Shao, Shalaby MR, et al (2006) Cancer Res 66: 2801-2806. Link: https://tinyurl.com/ycnhhno7

113. www.nanotechwire.com. Link: https://tinyurl.com/odx97yg

114. Mehta A (2009) Pharmaceutical Science Master's Theses, Northeastern University. Link: https://tinyurl.com/yc4urav9

115. Drummond DC, Noble CO, Guo Z, Connolly-Ingrama C, Gabriela SB et al
(2010) J Control Release 141: 13-21. Link: https://tinyurl.com/ybtncksw

116. Basu S, Basu M. (2008) Liposome methods and protocols 199. Link: https://tinyurl.com/y88yeo4x

117. Link: https://tinyurl.com/y9m7gg9c

118. Hope MJ, Bally MB, Webb G, Cullis PR (1985) Production of large unilamellar vesicles by a rapid extrusion procedure: characterization of size distribution, trapped volume and ability to maintain a membrane potential. Biochim Biophys Acta 812: 55-65. Link: https://tinyurl.com/ycc47m4u

Copyright: (C) 2018 Rahman A, et al. This is an open-access article distributed under the terms of the Creative Commons Attribution License, which permits unrestricted use, distribution, and $r$ eproduction in any medium, provided the original author and source are credited. 